\title{
Hitting more birds with one stone: CD70 as an actionable immunotherapeutic target in recurrent glioblastoma
}

Seyfrid $\mathrm{M}^{{ }^{*}}$, Maich $\mathrm{W}^{2^{*}}$, Shaikh $\mathrm{MV}^{1}$, Tatari $\mathrm{N}^{2}$, Upreti $\mathrm{D}^{1}$, Piyasena $\mathrm{D}^{2}$, Subapanditha $\mathrm{M}^{1}$, Savage $\mathrm{N}^{2}$, McKenna $\mathrm{D}^{1}$, Kuhlmann $\mathrm{L}^{3}$, Khoo $\mathrm{A}^{3}$, Salim SK${ }^{2}$, Bassey-Archibong $\mathrm{B}^{1}$, Gwynne W ${ }^{1}$, Chokshi $C^{2}$, Brown $\mathrm{K}^{4}$, Murtaza N², Bakhshinyan $\mathrm{D}^{2}$, Vora $\mathrm{P}^{1}$, Venugopal $\mathrm{C}^{1}$, Moffat $\mathrm{J}^{4}$, Singh $\mathrm{SK}^{1,2}$.

*These authors made equal contributions to this paper

Departments of ${ }^{1}$ Surgery, ${ }^{2}$ Biochemistry and Biomedical Sciences, Faculty of Health Sciences, McMaster University, 1200 Main Street West, Hamilton, Ontario, L8N 3Z5, Canada

${ }^{3}$ Princess Margaret's Hospital, Toronto, Ontario, M5S 3E1, Canada

${ }^{4}$ Donnelly Centre, Department of Molecular Genetics, University of Toronto, Ontario, Canada

\section{RUNNING TITLE}

CD70 as an immunotherapeutic for recurrent GBM

KEYWORDS: Glioblastoma, CD70, Immunotherapy, CAR-T

\section{CONFLICTS OF INTEREST:}

This work was partly funded by Longbow Therapeutics (company no longer in existence) 


\section{TRANSLATIONAL RELEVANCE}

Glioblastoma is the most common adult malignant brain tumor, and is characterized by a dismal prognosis and poor response to therapy at recurrence. Little-to-no change in standard of care therapy in the last 15 years, despite numerous potential therapies entering clinical trials.

Therapeutic failure is largely due to tumor heterogeneity, and a lack of unique tumor associated antigens. Here, we propose CD70 as an immunotherapeutic target in recurrent glioblastoma. CD70 plays a role in key pro-tumorigenic processes and is minimally expressed in normal tissues. We develop a CD70-directed CAR-T cell, which we show to be highly efficacious in extending survival in our intracranial mouse models of recurrent GBM. In addition, we identify CD27 - the receptor for CD70 - on the surface of multiple populations within the tumor immune microenvironment, implying that CD70/CD27 interactions may play a role in the tumor immune microenvironment. 


\begin{abstract}
:
Purpose: Glioblastoma (GBM) patients suffer from a dismal prognosis, with standard of care therapy inevitably leading to therapy-resistant recurrent tumors. The presence of brain tumor initiating cells (BTICs) drives the extensive heterogeneity seen in GBM, prompting the need for novel therapies specifically targeting this subset of tumor-driving cells. Here we identify CD70 as a potential therapeutic target for recurrent GBM BTICs.
\end{abstract}

Experimental Design: In the current study, we identified the relevance and functional influence of CD70 on primary and recurrent GBM cells, and further define its function using established stem cell assays. We utilize CD70 knockdown studies, subsequent RNAseq pathway analysis, and in vivo xenotransplantation to validate CD70's role in GBM. Next, we developed and tested an anti-CD70 CAR-T therapy, which we validated in vitro and in vivo using our established preclinical model of human GBM. Lastly, we explored the importance of CD70 in the tumor immune microenvironment (TIME) by assessing the presence of its receptor, CD27, in immune infiltrates derived from freshly resected GBM tumor samples.

Results: CD70 expression is elevated in recurrent GBM and CD70 knockdown reduces tumorigenicity in vitro and in vivo. CD70 CAR-T therapy significantly improves prognosis in vivo. We also found CD27 to be present on the cell surface of multiple relevant GBM TIME cell populations.

Conclusion: CD70 plays a key role in recurrent GBM cell aggressiveness and maintenance. Immunotherapeutic targeting of CD70 significantly improves survival in animal models and the CD70/CD27 axis may be a viable poly-therapeutic avenue to cotarget both GBM and its TIME.

\title{
ACKNOWLEDGMENTS
}

M.S. was supported by MITACS fellowship along with Longbow therapeutics. S.K.S. is supported by the Terry Fox Program Project grant and McMaster University Department of Surgery. We thank Dr. Mary Haak-Frendscho and Dr.Alan Wahl for providing us with CD70 antibodies.

\section{CORRESPONDING AUTHOR}

Sheila K. Singh, MD, PhD, FRCS(C)

Associate Professor of Pediatric Neurosurgery, Department of Surgery, McMaster Children's Hospital, Scientist, McMaster Stem Cell \& Cancer Research Institute MDCL 5027, 1280 Main Street West, Hamilton, ON, L8S 4K1, Canada

T: 905-521-2100 x75237 F: 905-521-992 Email: ssingh@mcmaster.ca 


\section{INTRODUCTION}

Glioblastoma (GBM) is the most common malignant brain tumor in adults accounting for approximately $14.6 \%$ of all brain tumors (Ostrom et al. 2019). Despite an aggressive standard of care (SoC) including maximal surgical resection and chemoradiotherapy, GBM patients have a median survival time of less than 15 months, and a five-year survival rate of less than 6.8\% (Stupp et al. 2005; Stupp et al. 2017; Ronning et al. 2012). GBM often recurs 7-9 months after resection of the primary tumor, at which point the tumor is often non-resectable, and poorly responsive to chemo- and/or radiotherapy, leaving patients with therapeutic options limited to clinical trial enrollment (van Linde, M. E., 2017).

In the past three decades, survival rates across several cancers have improved significantly, due in part to major advances in technology allowing for early detection, as well as significant leaps in targeted and novel therapeutic strategies (Siegel, Miller, and Jemal 2018). However, despite these advances, little to no improvement has been made in prognosis for GBM patients, who continue to suffer from dismal outcomes.

Therapeutic failure, in part, is due to extensive intratumoral heterogeneity at the cellular, genetic, and functional levels (Bergmann et al. 2020; Soeda et al. 2015; Xiong, Yang and Li 2020). This heterogeneity may be explained by a distinct subset of cells coined brain tumor initiating cells (BTICs) (Soeda et al. 2015), which possess stem celllike traits such as self-renewal, therapy evasion, and multi-lineage differentiation (Cusulin et al. 2015). It is believed that this subpopulation of BTICs, after undergoing selective pressures from primary GBM SoC therapy, become chemo- and radio-therapy resistant, and seed formation of the therapy-resistant recurrent tumors (Liu et al. 2006; Bao et al. 2006). Expression of GBM BTIC markers such as CD133, CD15, and CD44 are generally associated with worse clinical outcome (Zeppernick et al. 2008). Thus, novel therapeutic interventions to target not only the tumor bulk, but the treatment resistant BTIC population that seeds recurrence is necessary.

Immunotherapy holds great promise in cancer treatment, and recent studies in gliomas provide encouraging results (Vora et al. 2020; Morgan et al. 2012; Jin et al. 2018). Amongst various immunotherapeutic approaches are adoptive T cell therapies, including chimeric antigen receptor (CAR) T-therapy. CAR-Ts are T-cells expressing a recombinant 
cell-surface receptor that directs these cells to specific tumor associated antigens (TAAs). Upon binding to the TAA, T-cells undergo MHC-independent activation and induce apoptosis of the target cell (Priceman, Forman, and Brown 2015; Zhang et al. 2017). However, to develop safe and effective CAR-T cells, novel tumor-specific antigens with a sufficient therapeutic window are required.

Genomic and proteomic data from a multi-omic target development pipeline revealed CD70 as a suitable therapeutic target in recurrent GBM (rGBM). Our data shows that CD70 is more highly expressed in rGBM samples compared to primary GBM (pGBM) samples. Moreover, CD70 is absent on normal human astrocytes and neural stem cells, as is supported by the literature (Grewal et al. 2008), thereby presenting a novel opportunity to target recurrent GBM. CD70 is a transmembrane glycoprotein and a member of the tumor necrosis factor (TNF) superfamily, and is the only known ligand for CD27. While CD70 is transiently expressed on activated T- B-cells, as well as mature dendritic cells, it is minimally expressed in most normal tissues (Jacobs et al. 2015, Adam P.J. et al. 2006). Similarly, CD27 is primarily only expressed on specific subsets of T- B-, and NK cells (Bowman et al. 1994; Hintzen et al. 1994; Tesselaar et al. 2003). The CD70/CD27 signalling axis leads to differentiation, proliferation, and T- and B-cell survival and proliferation (Denoeud and Moser 2011; Nolte et al. 2009; Borst, Hendriks, and Xiao 2005). Prolonged expression of CD70 has been shown to elicit lethal immunosuppression in mice (Tesselaar et al. 2003), and result in exhaustion of effector memory T-cells in Bcell non-Hodgkin lymphoma (Yang et al. 2014).

CD70 displays aberrant constitutive expression in a variety of cancers, include renal cell carcinoma, leukemia, non-small cell lung cancer, melanoma, GBM, and others (Diegmann et al. 2005; Lens et al. 1999; Jacobs et al. 2015; Pich et al. 2016; Held-Feindt and Mentlein 2002; Hishima et al. 2000; Pahl et al. 2015). In 2005, researchers showed that in B-cell lymphoma, CD70 and CD27 are mutually overexpressed, resulting in increased proliferation and survival of tumor cells via amplified signalling through the CD70/CD27 axis (Nilsson et al. 2005). In the context of GBM, CD70 has been shown to promote tumor progression and invasion (Ge et al. 2017). While in healthy individuals CD70 plays a role in eliciting an immune response, its role in the tumor microenvironment is far more multi-faceted. Within the GBM microenvironment, CD70 mediates immune 
escape (Wischhusen et al. 2002), and its overexpression leads to recruitment and activation of immunosuppressive T regulatory cells (Tregs) (Claus et al. 2012) and tumor associated macrophages (TAMs) (Ge et al. 2017). Together, these studies suggest that CD70 plays a major role in the recruitment and maintenance of the GBM immunosuppressive microenvironment, while promoting pro-tumorigenic processes.

The soluble form of CD27 (sCD27) is detected at high levels in the blood of cancer patients (Purdue et al. 2019; Kashima et al. 2019). Currently, there are multiple therapeutic strategies targeting CD70-expressing malignancies (Aftimos et al. 2017; Bristol-Myers Squibb 2013; Seagen Inc. 2018), however, the prevalence of the CD70/CD27 interaction provides a rationale for synergistic therapeutic opportunities targeting both tumor cells and the immune microenvironment.

To our knowledge, this is the first time CD70 has been identified as an immunotherapeutic target on BTICs from patient-derived GBM samples. In the presented work, we conduct a systematic study evaluating the efficacy of CD70 CAR-T cells in using our established patient-derived GBM mouse model, illustrating the potential of a CD70directed CAR-T therapy to offer hope to GBM patients suffering from a dismal prognosis.

\section{Material and methods}

\section{Dissociation and culture of primary GBM tissue}

Human GBM samples (Table S1) were obtained from consenting patients, as approved by the Hamilton Health Sciences/McMaster Health Sciences Research Ethics Board. Brain tumor samples were dissociated in PBS (ThermoFisher, Cat\#10010049) containing 0.2 Wunsch unit/mL Liberase Blendzyme 3 (Millipore Sigma, Cat\#5401119001), and incubated in a shaker at $37^{\circ} \mathrm{C}$ for $15 \mathrm{~min}$. The dissociated tissue was filtered through a

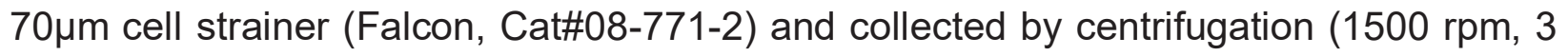
min). Red blood cells were lysed using ammonium chloride solution (STEMCELL Technologies, Cat\#07850). GBM cells were resuspended in Neurocult complete (NCC) media, a chemically defined serum-free neural stem cell medium (STEMCELL Technologies, Cat\#05751), supplemented with human recombinant epidermal growth factor (20ng/mL: STEMCELL Technologies, Cat\#78006), basic fibroblast growth factor (20ng/mL; STEMCELL Technologies Cat\#78006), heparin (2 mg/mL 0.2\% Heparin 
Sodium Salt in PBS; STEMCELL technologies, Cat\#07980), antibiotic-antimycotic (1X; Wisent, Cat\# 450-115-EL), and plated on ultra-low attachment plates (Corning, Cat\#431110) and cultured as neurospheres. GBM8 and GBM4 was a kind gift from Dr. Hiroaki Wakimoto (Massachusetts General Hospital, Boston, MA, USA), RN1, S2b2 and WK1 were gifts from Dr Andrew Boyd (QIMR Berghofer Medical Research Institute, Australia).

\section{Propagation of Brain tumor stem cells (BTSCs)}

Neurospheres derived from minimally cultured (<20 passages) human GBM samples were plated on polyornithine- laminin coated plates for adherent growth. Adherent cells were replated in low-binding plates and cultured as tumorspheres, which were maintained as spheres upon serial passaging in vitro. As shown before, compared to commercially available GBM cell lines, patient derived 3D cultures represent the variety of heterogeneous clones present within patient samples (Patrizii et al. 2018). These models recapitulate the key GBM morphological, architectural and expression features that are present in primary GBM. These cells retained their self-renewal potential and were capable of in vivo tumor formation.

\section{Glycocapture Proteomics}

Briefly, cells were lysed in PBS:TFE (50:50) using pulse sonication and by incubating the lysates at $60^{\circ} \mathrm{C}$ for 2 hours (lysates were vortexed every 30 minutes). Protein concentration was determined using the BCA assay (Pierce). Cysteines were reduced with DTT ( $5 \mathrm{mM}$ final concentration) at $60^{\circ} \mathrm{C}$ for 30 minutes and alkylation was performed by adding iodoacetamide $(25 \mathrm{mM}$ final concentration) to the cooled lysates and subsequent incubation at room temperature for 30 minutes. Trypsin was added at a 1:500 ration and protein digestion was performed overnight at $37^{\circ} \mathrm{C}$. Tryptic peptides were desalted on C18 Macrospin columns (Nest Group), lyophilized and resuspended in coupling buffer ( $0.1 \mathrm{M}$ Sodium Acetate, $0.15 \mathrm{M}$ Sodium Chloride, $\mathrm{pH} 5.5$ ). Glycan chains were oxidized using $10 \mathrm{mM} \mathrm{NaIO}_{4}$ for 30 minutes in the dark and peptides were again desalted. Lyophilized peptides were resolubilized in coupling buffer and oxidized glycopeptides were captured on hydrazide magnetic beads 
(Chemicel, SiMAG Hydrazide) for $12 \mathrm{~h}$ at room temperature. The coupling reaction was catalysed by adding aniline $(50 \mathrm{mM})$ and the reaction was allowed to continue for additionally 3 hours at room temperature.

Hydrazide beads containing the covalently coupled oxidized glycopeptides were thoroughly washed (2 x coupling buffer; $5 x 1.5 \mathrm{M} \mathrm{NaCl}$; $5 x$ HPLC H2O; 5x Methanol; 5x $80 \%$ Acetonitrile; 3x Water; $3 \times 100 \mathrm{mM} \mathrm{NH}_{4} \mathrm{OH}, \mathrm{pH} 8.0$ ) to remove non-specific binders. $\mathrm{N}$-glycopeptides were eluted off the hydrazide beads using $5 U$ PNGase F in 100mM ammonium bicarbonate at $37^{\circ} \mathrm{C}$ overnight. The de-glycosylation reaction converts the asparagine residue, covalently linked to a glycan chain, to aspartic acid, the process carrying a signature mass shift of $0.98 \mathrm{Da}$.

Eluted (i.e. deamidated) glycopeptides were recovered and the hydrazide beads were additionally washed 2 X with $80 \%$ acetonitrile solution. Glycopeptides were desalted using C18 stage tips, eluted using $80 \%$ acetonitrile, 0.1\% F.A. and lyophilised. The purified glycopeptides were dissolved in $21 \mu \mathrm{L} 3 \%$ acetonitrile, $0.1 \%$ F.A. Peptide concentration was determined using a NanoDrop 2000 (Thermo) spectrophotometer.

\section{RNA sequencing and GSEA/Cytoscape analysis}

Total RNA was extracted using the Norgen Total RNA isolation kit (Cat \#48400) and quantified using a NanoDrop Spectrophotometer ND-1000. The RNA was sequenced using single-end 50 bp reads on the Illumina HiSeq platform (Illumina, San Diego CA, USA). Raw sequence data were exported to FASTQ format and were filtered based on quality scores (Quality cutoff of 20 for at least $90 \%$ of the bases in the sequence). Next the reads were mapped to the UCSC mRNA transcript human database based on the GRCh38/hg38 version using HISAT. The counts were obtained by using htseq count with the "intersection-strict" option. Counts were transformed with TMM transformation and then normalized with VOOM (package "limma" in R).

Differential gene expression profiles were generated by DESeq2 using the Galaxy online suite (https://usegalaxy.org/) and as imput of the Gene Set Enrichment Analysis (GSEA). Gene sets were randomized at 2000 permutations per analysis against Oncogenic (C6), Curated (C2) and Hallmark MSigDB collections of gene sets (https://www.gsea-msigdb.org/gsea/msigdb/index.jsp). 


\section{Secondary sphere formation assay}

Tumorspheres were dissociated using 5-10 $\mu \mathrm{L}$ Liberase Blendzyme3 (0.2 Wunsch unit $/ \mathrm{mL}$ ) in $1 \mathrm{~mL}$ PBS for $5 \mathrm{~min}$ at $37^{\circ} \mathrm{C}$. Based on each cell line's growth kinetics, cells were plated at 200-1000 cells per well in $200 \mu \mathrm{L}$ of NCC media in a 96-well plate. Cultures were left undisturbed at $37^{\circ} \mathrm{C}, 5 \% \mathrm{CO} 2$. After four days, the number of secondary spheres formed were counted.

\section{Cell proliferation assay}

Single cells were plated in a 96-well plate at a density of 200-1000 cells/200 $\mu \mathrm{L}$ (based on each cell line's growth kinetics) per well in quadruplicate and incubated for five days. $20 \mu \mathrm{L}$ of Presto Blue (ThermoFisher, Cat\#A13262), a fluorescent cell metabolism indicator, was added to each well approximately $4 \mathrm{~h}$ prior to the readout time point. Fluorescence was measured using a FLUOstar Omega Fluorescence 556 Microplate reader (BMG LABTECH) at excitation and emission wavelengths of $535 \mathrm{~nm}$ and $600 \mathrm{~nm}$ respectively. Readings were analyzed using Omega analysis software.

\section{Receptor internalization and Antibody Drug Conjugate assay}

For detection of internalization, 200,000 cells were being used for each condition, where both were incubated with antibody $30 \mathrm{~min}$ on ice, rinsed twice and let incubated for $2 \mathrm{~h}$ either at $37^{\circ} \mathrm{C}$ or at $4 \mathrm{C}$, before being analyzed under flow cytometry.

GBM BTICs expressing CD70 on their cell surface were seeded and incubated for 30 minutes with different concentrations of he-Im-Fab'2 anti-CD70, followed by addition of $13 \mathrm{nM}$ of $2^{\circ} \mathrm{ADC} \alpha$-HFab-NC-MMAF (conjugated with Monomethyl auristatin F) (Moradec, Cat\# AH-121-AF) and proliferation was measured after 5 days ( $n=3$ for BT241s, $n=2$ for HEK293s and $n=1$ for BT935s). The manufacturer's protocol was followed directly, with the exception of using twice the initial amount of recommended antibody $(40 \mathrm{nM})$.

\section{In vivo intracranial injections and H\&E/immunostaining of xenograft tumors}

Animal studies were performed according to guidelines under Animal Use Protocols of McMaster University Central Animal Facility. Intracranial injections in 6-8 week-old NSG 
mice were performed as previously described (Singh et al. 2004) using either BT241, GBM8 or GBM4 cells (100,000 cells/mice). Briefly, a burr hole is drilled at the point located $2 \mathrm{~mm}$ behind the coronal suture, and $3 \mathrm{~mm}$ to the right of the sagittal suture and GBM cells suspended in $10 \mu \mathrm{L}$ PBS are intracranially injected with a Hamilton syringe (Hamilton, Cat\#7635-01) into right frontal lobes of 6-8 week-old NSG mice. For CAR-T treatment, ConCAR-T or CD70CAR-T cells were injected intratumorally once a week for two weeks (for BT241, 1M first week then 0.5M; for GBM8 0.75M first week then 1M for GBM8). For tumor volume evaluation, animals were sacrificed when control mice reached endpoint. When mice reached endpoint, they were perfused with $10 \%$ formalin and collected brains were sliced at $2 \mathrm{~mm}$ thickness using brain-slicing matrix for paraffin embedding and H\&E staining. Images were captured using an Aperio Slide Scanner (Leica Biosystems) and analyzed using ImageScope v11.1.2.760 software (Aperio). For survival studies, all the mice were kept until they reached endpoint and number of days of survival were noted for Kaplan Meyer Analysis. CD3 stained slides were scanned and captured using an Aperio Slide Scanner and analyzed using ImageScope v11.1.2.760 software (Aperio). Tumor areas were generated using Aperio Membrane Algorithm.

\section{Generation of CAR Lentivirus}

Human anti-CD70 (he_I and he_Im) scFv sequence were synthesized with a 5' leader sequence and 3' Myc tag by Genescript. The scFv was cloned into the lentiviral vector pCCL $\triangle$ NGFR (kindly provided by Dr. Bramson, McMaster University, Hamilton, ON, Canada) down- stream of the human EF1a promoter leaving $\triangle N G F R$ intact downstream of the minimal cytomegalovirus promoter. Empty pCCL $\triangle N G F R$ was used as a control vector. Replication-incompetent lentiviruses were produced by cotransfection of the CAR vectors and packaging vectors pMD2G and psPAX2 in HEK293FT cells using Lipofectamine 3000 (ThermoFisher, Cat\#L3000075) as recommended by the manufacturer. Viral supernatants were harvested 24 and 48 hours after transfection and concentrated by ultracentrifugation at 15,000 RPM for $2 \mathrm{~h}$ at $4^{\circ} \mathrm{C}$. The viral pellet was resuspended in $1.0 \mathrm{~mL}$ of $\mathrm{T}$ cell media, aliquoted and stored at $-80^{\circ} \mathrm{C}$. 


\section{Generation of CAR-T cells}

Peripheral blood mononuclear cells (PBMCs) from consenting healthy blood donors were obtained using SepMate ${ }^{\mathrm{TM}}$ (STEMCELL technologies, Cat\#85450). This research was approved by the McMaster Health Sciences Research Ethics Board. $1 \times 10^{5}$ cells in XSFM media (Irvine Scientific, Cat\#91141) were activated with anti-CD3/CD28 beads at a 1:1 ratio (Dynabeads, GIBCO, Cat\#113.31D) in a 96-well round bottom plate with $100 \mathrm{U} / \mathrm{mL}$ rhIL-2 (Peprotech, Cat\#200-02). Twenty-four hours after activation, T cells were transduced with lentivirus at a MOI 1. CAR-T cell cultures were expanded into fresh media (XSFM media supplemented with $100 \mathrm{U} / \mathrm{mL}$ rhIL-2) as required for a period of 6-8 days prior to experimentation.

\section{Evaluation of cytokine release}

NGFR+ sorted CAR-T cells (CD70CAR or ConCAR) were co-incubated with GBM cells at a 1:1 ratio for 24 hours. Supernatants were collected in duplicate for each condition and stored at $80^{\circ} \mathrm{C}$ for analysis of cytokines. Human TNF- $\alpha$ DuoSet ELISA kit (R \& D Systems, Cat \#: DY210-05) and IFN-y DuoSet ELISA kit (R \& D Systems, Cat \#: DY285B05) were used for quantification of the two cytokines by ELISA, according to manufacturer's recommendation.

\section{Flow cytometric analysis and sorting}

GBM cells and T cells in single cell suspensions were resuspended in PBS+2mM EDTA. GBM cells were stained with he_l or he_Im IgG $(0.064-1000 \mathrm{nM})$ or by IgG control AffiniPure Goat Anti-Human IgG, $F\left(a b^{\prime}\right) 2$ fragment specific, Jackson ImmunoResearch, Cat\#109-005-006) or APC-conjugated anti-CD70 antibody (Miltenyi Biotech, REA 292) and incubated for $30 \mathrm{~min}$ on ice. CAR-T cells were stained with fluorescent tagged anti-CD3 (BD Biosciences, Cat\#557851), anti-NGFR (Miltenyi Biotech, Cat\#130-112-790) and anti-c-Myc (Miltenyi Biotech, Cat\#130-116653). Samples were run on a MoFlo XDP Cell Sorter (Beckman Coulter). Dead cells were excluded using the viability dye 7AAD (1:10; Beckman Coulter, A07704). Compensation was performed using mouse IgG CompBeads (BD Biosciences, Cat\#552843). 


\section{Cytotoxicity assays}

Luciferase-expressing GBM cells at a concentration of 30,000 cells/well were plated in 96-well plates in triplicates. In order to establish the BLI baseline reading and to ensure equal distribution of target cells, D-firefly luciferin potassium salt $(15 \mathrm{mg} / \mathrm{mL})$ was added to the wells and measured with a luminometer (Omega). Subsequently, effector cells were added at 4:1, 3:1, 2:1, 1:1, and 0:1 effector-to- target $(\mathrm{E}: \mathrm{T})$ ratios and incubated at $37^{\circ} \mathrm{C}$ for 4-8 hours. BLI was then measured for $10 \mathrm{~s}$ with a luminometer as relative luminescence units $(\mathrm{RLU})$. Cells were treated with $1 \%$ Nonidet P-40 (NP40, Thermofisher, Cat\#98379) to measure maximal lysis. Target cells incubated without effector cells were used to measure spontaneous death RLU. The readings from triplicates were averaged and percent lysis was calculated with the following equation:

$\%$ Specific lysis $=100$ (spontaneous death $R L U-$ test $R L U$ )(spontaneous death $R L U--m$ aximal killing $R L U) \%$

Specific lysis=100(spontaneous death RLU- test RLU)(spontaneous death RLU--maxi mal killing RLU)

\section{Isolation and evaluation of immune cells from Brain tumor samples}

EasySep human CD45 Depletion kit II (Stem Cell Technology, Cat\#: 17898) was used to extract immune cells from freshly dissected patient tumors, according to the manufacturer's protocol but with slight modifications. Briefly:

1. Prepare a single-cell suspension from tumor like before and resuspend the cells at 10^8cells/mL in EasySep Buffer (Cat \#: 20144) (If the tumor is too small, and total cells are less than $10^{\wedge} 7$ cells resuspend in $0.1 \mathrm{ml}$ and adjust antibody and reagents (step 2 and 4 accordingly):

2. Add $12.5 \mu \mathrm{L} / \mathrm{mL}$ of EasySep Human CD45 Depletion Cocktail II (Component\#17898C)

3. Incubate $5 \mathrm{~min}$ at RT

4. Add $20 \mu \mathrm{L} / \mathrm{mL}$ of EasySep ${ }^{\mathrm{TM}}$ Dextran RapidSpheres 50101 (Component \#50101)

5. Incubate $3 \min$ at RT 
6. Top up volume (EasySep Buffer) to $2.5 \mathrm{ml}$ and place tube into the EasySep magnet (Cat \#: 18000) for 5 minutes

7. Pour off CD45- cells - cells remaining in tube are CD45+ population

8. For optimal recovery, perform $2 \times 5$ min separation in the magnet

To identify individual tumor immune microenvironment immune cell populations, CD45+ cells were thawed and used immediately to run a panel of antibodies in order to identify individual immune cell populations. Antibodies used are as follows and were used according to manufacturer's protocol: PE-Cy7 Mouse Anti-Human CD3 (Cat 563423; BD Pharmingen), PE Mouse Anti-Human CD4 (Cat555347; BD Pharmingen), APC Mouse Anti-Human CD8 (Cat555369; BD Pharmingen), PE-CF594 Mouse Anti-Human CD68 (Cat564944; BD Horizon), APC-H7 Mouse Anti-Human HLADR (Cat561358; BD Pharmingen), BV421 Mouse Anti-Human CD27 (Cat562514; BD Horizon).

\section{CAR-T Fratricide}

Jurkat human T lymphocytes (Cedarlane CaA\#: TIB-152) were expanded and grown in RPMI 1640 (Gibco Cat\#:11875-093) with 10\% FBS (Multicell Cat\#:08105), 1\% PenicillinStreptomycin (Gibco Cat\#:15140-122) and 10 mM HEPES (Gibco Cat\#: 15630080). Jurkat cells were then transduced with either an shGFP or shCD70 lentiviral construct and selected for by puromycin selection. shGFP Jurkat cells were sorted by flow cytometry to isolate a CD70 hi shGFP population, and untransduced Jurkat cells were sorted to isolate a CD70 hi population. shGFP and shCD70 Jurkat cells were then transduced with either Control CAR or CD70 CAR virus and allowed to expand. At 4 and 8 days after transduction, each population (shGFP ConCAR; shGFP CD70CAR; shCD70 ConCAR; shCD70 CD70CAR) was assessed by flow cytometry for the following markers: NGFR, CD70, CD69, viability.

\section{Statistical Analysis}

Biological replicates from at least three patient samples were compiled for each experiment, unless otherwise specified in figure legends. Respective data represent 
mean $\pm \mathrm{SD}, n$ values are listed in figure legends. Student's $t$ test analyses were performed using GraphPad Prism 5. $p>0.05=$ n.s., $p<0.05={ }^{*}, p<0.01={ }^{* *}, p<0.001={ }^{* *}, p<$ $0.0001=* * * *$.

\section{RESULTS}

\section{CD70 expression is a unique marker of recurrent glioblastoma}

Between the underrepresentation of rGBM samples in biobanks, due to the relatively low re-operation rate at GBM recurrence, and the variable presence of BTICs within bulk tumor samples, rGBM targets are often overlooked (Robin, Lee, and Kalkanis 2017). In this study, we leveraged an RNA sequencing platform using four in-house, lowpassage BTIC-enriched cell lines derived from pGBM or rGBM patient samples, as previously described by our lab (Venugopal et al. 2012). Using the GBM TCGA repository (Bowman et al. 2017) we identified genes over-represented in BTIC-enriched populations, among which we found the TNF superfamily member CD70, which was present in pGBM BTICs but particularly upregulated in rGBM BTICs. (Fig1A, SupplFig1A). To further investigate the relevance of $\mathrm{CD} 70$ as a recurrent GBM marker, we used six primary/recurrent pairs from patient-matched GBM samples present in the TCGA database to evaluate CD70 expression. In silico analysis of CD70 mRNA expression revealed increased levels in rGBM samples compared to their matched primaries for the majority of the pairs available, however this trend did not reach significance (Fig1B). Additionally, these same matched pairs exhibited a Classical (TCGA-CL) to Mesenchymal (TCGA-MES) subtype transition from primary to recurrence, indicating a shift towards a more aggressive and therapy-resistant subtype with poorer prognosis (Wang et al. 2017, Sa et al. 2020). Given that mRNA expression does not necessarily translate directly to cell-surface protein expression, we interrogated cell-surface CD70 protein levels on two in-house matched primary/recurrent patient derived BTIC lines. We observed an increase in CD70 surface expression in both pairs by flow analysis (Fig1C) and a switch from the CL to MES subtype as seen in our bulk RNA sequencing samples (BT594/BT972, data not shown). We next screened a variety of unmatched primary and recurrent GBMs, as well as normal human cells lines (neural stem cells and astrocytes) for CD70 expression (Fig1D, SupplFig1B). We identified a therapeutic window with normal human brain cells, which minimally express CD70 on their cell surface, in accordance with the existing 
literature (Hintzen et al. 1994). We demonstrate a clear trend towards increased CD70 expression in rGBM compared to pGBM (Fig1C), which bulk tumor data corroborates (Rahman et al. 2018). Lastly, using our BT594/BT972 matched-pair, we performed NGlycocapture Proteomics which ranked CD70 among the top upregulated cell surface markers in rGBM compared to pGBM (Fig1E). This data led us to further inquiries about the functional role that CD70 plays in GBM progression and maintenance.

\section{CD70 is a key player in GBM maintenance and tumor formation}

Given the upregulation of CD70 in GBM, specifically in rGBM, we sought to explore the role that CD70 expression plays in GBM maintenance and progression. We sorted pGBM and rGBM cells as CD70-positive or -negative using FACS analysis and carried out a PrestoBlue proliferation assay. CD70-positive cells demonstrated a significantly increased proliferation capacity compared to their CD70-negative counterparts (Fig2A, SuppIFig2A). We next aimed to assess the role of CD70 in sphere formation, a stem-like trait that is typical of BTICs and correlates with self-renewal capacity in vitro and tumorigenesis in vivo (Hirschhaeuser et al. 2010, Singh et al. 2004). In both CD70 ${ }^{H I G H}$ BTIC cell lines, silencing of CD70 using an shRNA knockdown vector led to a significant decrease in sphere formation capacity compared to controls (Fig2B). Given the correlation of sphere formation with tumorigenesis in vivo, we investigated whether CD70 silencing limits GBM tumor formation in our patient-derived orthotopic xenograft animal model. We generated CD70 knockdown (shCD70) and control lines (shGFP) of three GBM BTIC lines that naturally express high levels of CD70, and intracranially injected these into immunodeficient mice, as previously described (Singh et al. 2004). We observed a significant decrease in the size of tumors formed by shCD70 cells compared to shGFP controls, as determined by H\&E staining (Fig2C-D, SupplFig2C) and MRI imaging (Fig2G). This was further reflected in a significant survival advantage for mice engrafted with shCD70 cells compared to controls (Fig2E and F, SupplFig2D). These findings demonstrate that CD70 plays a key role in recurrent GBM proliferation, tumor formation and survival both in vitro and in vivo.

\section{CD70 plays a crucial role in cellular programs implicated in tumorigenesis}


Previous studies have emphasized the function of CD70 in GBM as it contributes to T-cell apoptosis, and mediates tumor cell migration and invasion, a feature characteristic of mesenchymal-like cells (Ge et al. 2017; Diegmann et al. 2006; Inaguma et al. 2020). To further investigate the role of the CD70 signaling network in GBM, we investigated transcriptional changes and their predicted networks after CD70 silencing using RNA sequencing and subsequent gene set enrichment analysis (GSEA). Using three GBM lines transduced with shCD70 or shGFP, we observed strong downregulation of FOSL1, a gene recently discovered to play a pivotal role in stemness, migration, and EMT (SupplFig3A) (Fiscon, Conte, and Paci, 2018; Feldker et al. 2020). Other slightly downregulated genes included CDH2 (Cadherin 2), PLAUR, and CXCR4; genes known to be associated with the Mesenchymal subtype in GBM and a worse overall prognosis (Gilder et al. 2018; Tao et al. 2020; Yi et al. 2018). OLIG2, a transcription factor commonly associated with the proneural subtype and tumor recurrence (Bouchart et al. 2020, Lu et al. 2016), showed upregulation following CD70 knockdown, while expression of the proangiogenic factor VEGFA was depressed, indicating that CD70 may play a role in GBM angiogenesis, a characteristic previously documented in other pathologies, but not in cancer (Simons et al. 2018, Winkels et al. 2017).

GSEA was performed using Gene Ontology (Merico et al. 2010) and MSigDB C2 and C6 gene sets (Subramanian et al. 2005; Liberzon et al. 2011), to gain a deeper understanding of the cellular programs associated with CD70 expression (SupplFig3A). Top modulated pathways showed that silencing CD70 results in downregulation of epithelial-to-mesenchymal transition (EMT) and hypoxia signatures, and upregulation of Interferon type I/interleukin-1 pro-inflammatory signatures (Guarda et al. 2011) (SupplFig3A, B). Hypoxia and EMT pose major hurdles in GBM, as they promote migration of tumor cells further into the brain tissue, while pro-inflammatory signals are often depressed in GBM (Monteiro et al. 2017; Carro et al. 2010; Singh, A, et al. 2010). While these data are limited, they do further implicate the role of CD70 in various processes linked to invasiveness, immunosuppression, and poor prognosis in GBM, as well as angiogenesis and stem-like characteristics of GBM BTICs.

\section{Generation and characterization of CD70-directed CAR T cells}


Adoptive cell therapies have shown great promise in overcoming therapy resistance and providing a more specific targeted therapy in multiple cancers, including in GBM (Bielamowicz, Khawja, and Ahmed, 2013). However, despite significant global efforts to develop these therapies, they have only been approved for B cell malignancies thus far, and have yet to show efficacy in solid tumors such as GBM (O'Rourke et al. 2017). It is believed that this lack of progress is in part due to the immunosuppressive microenvironment of solid tumors, particularly GBM, as well as antigen escape (Sterner and Sterner, 2021).

Given our data implicating CD70 as a key factor in GBM functionality, we tested two distinct in-house fragments antigen-binding (Fabs) for their ability to bind cell-surface CD70, and compared these to commercially available CD70 antibody (Fig4A). The Fab he-Im was used to develop a non-covalently conjugated therapeutic antibody-drug conjugate (ADC) (Fig4B), which we postulated would be advantageous due to the rapid internalization of CD70 upon ligand binding (Adam et al. 2006; McDonagh et al. 2008) (SupplFig4A). We incubated CD70 ${ }^{\mathrm{HIGH}}$ GBM cells for 72 hours with our ADC, and observed a dramatic cytotoxic effect; an effect not seen in CD70 LOW GBM cells or HEK293 control cells, indicating that our ADC is both specific and cytotoxic, and is suitable for developing adoptive cell therapies. Thus, we cloned the scFv region of he-Im into a second-generation CAR linked to a truncated c-Myc tag (Fig4C), and achieved moderate CAR cell-surface expression nine days post-transduction in human T-cells (Fig4D). To determine the efficacy of these anti-CD70 CAR-T (CD70CAR-T) cells, we co-cultured them with $\mathrm{CD} 70^{\mathrm{HIGH}}$ GBM cells. CD70CAR-T cells co-cultured with CD70 ${ }^{\mathrm{HIGH}}$ GBM cells released significantly more IFN-Y and TNF- $\alpha$ into culture supernatants compared to a control CAR-T (ConCAR-T) (Fig4E, SupplFig 4B, C). Additionally, CD70CAR-Ts demonstrated significant cytotoxicity against numerous CD70 ${ }^{\mathrm{HIGH}}$ GBM cells at effector to target ratios as low as 1:1 (Fig4F, SupplFig4D). Together, these data indicate that CD70CAR-T cells are capable of mounting a robust and specific immune response against CD70-expressing GBMs.

Lastly, we assessed the antitumor potential of our CD70CAR-T cells in orthotopically xenografted NODSCID mice, using CD70 ${ }^{\mathrm{HIGH}}$ BT241 rGBM BTICs. After confirming tumor engraftment using the In Vivo Imaging System (IVIS), we intracranially 
injected 1M CD70CAR-T or ConCAR-T cells weekly over two weeks. Mice treated with CD70CAR-T cells displayed significantly lower tumor burden, as observed by bioluminescence signal, and a significant decrease in tumor volume as shown by H\&E staining, confirming that CD70CAR-T cell-treated mice experience far less tumor growth compared to controls (Fig4A and B). Unsurprisingly based on our previous data, CD70CAR-T cell-treated animals had a significant survival advantage compared to control mice (Fig4C, left panel). Of note is that fact that the majority of animals (5 out of 9) did not display any tumor-related symptoms post-treatment, nor did H\&E staining display any presence of tumor at the end of study (Fig4B, right panel). To further validate our CD70CAR-T cell therapy, we reproduced this with another GBM cell line, and observed similar results, indicating that this approach is efficacious in multiple $\mathrm{CD} 7 \mathrm{H}^{\mathrm{HGH}}$ cell lines (SupplFig5A-C).

\section{CD70 and its role in the GBM tumor immune microenvironment}

CD70 is the only known ligand for the receptor CD27, a TNF receptor superfamily member, and is known to trigger T cell apoptosis and induce exhaustion (Chahlavi et al. 2005), as well as recruit tumor associated macrophages (TAMs) to the GBM microenvironment, contributing to the immunosuppressive nature of GBM (Ge et al. 2017). However, as far as its role in immune system functionality, no evidence has been found to date indicating it is essential (Shaffer et al. 2011). Thus, we elected to investigate the interaction between CD70-expressing GBM cells and CD27-expressing T cells, to see whether there would be any observed effect on T cell viability (Wajant et al. 2016). Additionally, CD70 cleaved from the cell surface and present in the supernatant may act similarly to cell-surface CD70 (Rowley and Al-Shamkhani, 2004). We co-cultured CD70 ${ }^{\mathrm{HIGH}}$ BT241 cells with CD27+ T cells, and observed a decrease in CD27+ T cell populations, an effect that was not seen when co-culturing with CD70 knockdown BT241 cells, indicating that CD70/CD27 interaction between T cells and GBM cells may initiate apoptotic programs in T cells, adding to the immunosuppressive capacity of GBM, as previously observed (Q. J. Wang et al. 2012) (SupplFig6B). We then cultured CD27+ T cells with supernatant from $\mathrm{CD} 70^{\mathrm{HIGH}}$ GBM cells to observe whether soluble CD70 
cleaved into the supernatant could bind CD27+ T cells and initiate downstream apoptotic effects, however, we observed no decrease in CD27+ T cell populations (SupplFig6B).

As seen in the literature (Lens et al. 1997), we observed increased expression of CD70 on activated T cells (Fig5C) and a subsequent decrease in CD70CAR-T cells count and viability, compared to ConCAR-T cells (data not shown). This insinuated that we were observing fratricide between CAR-T cells, as seen previously with other targets (SánchezMartínez et al. 2019). Thus, we elected to create a CD70 knockdown CD70CAR-T cell to overcome this problem. For this preliminary model we utilized Jurkat $\mathrm{T}$ cells due to their robustness compared to donor-derived T cells, and sorted them by flow cytometry to obtain a $\mathrm{CD} 70^{\mathrm{HIGH}}$ Jurkat cell population. We also created an shCD70 knockdown Jurkat cell population. Both $\mathrm{CD} 70^{\mathrm{HIGH}}$ and shCD70 Jurkats were transduced with CD70CAR construct or ConCAR construct, after which we examined any change in viability and presence of the activation marker CD69 (Fig5E). We were able to demonstrate that CD70 ${ }^{\mathrm{HIGH}}$ CD70CAR Jurkat cells had decreased viability and increased expression of the activation marker CD69, compared to both ConCAR and shCD70 CD70CAR Jurkats. This indicates that silencing of CD70 may be a viable option for overcoming CD70CAR T fratricide, improving viability of CD70CAR-T cells while having no effect on other T cell functions, as previously shown (Munitic et al. 2013, Kumar et al. 2020).

According to the literature, cancer cells may utilize the CD70/CD27 interaction to their advantage to modulate both $T$ cells and macrophages to create more immunosuppressive or immune-evasive environments (Ge et al. 2017; Inaguma et al. 2020). Due to the absence of an immune system in our immunodeficient mouse models, we elected to interrogate the GBM tumor immune microenvironment (TIME) using flow cytometry on CD45+ cells extracted from fresh patient-derived tumor samples. From this data we saw that CD27 had a similar expression pattern among non-T-cell populations (CD3- cells) from different tumor samples (Fig6A). Further, CD27 expression was found on putative 'M1 proinflammatory' (CD45+CD3-CD68+HLADR+) macrophages to a similar extent (Ma et al. 2010). It is interesting to note that CD27 expression was increased in this particular 'M1' population when their corresponding GBM cells were CD70 ${ }^{\text {HIGH }}$ (i.e., MBT190); a phenomenon not seen when correspond GBM cells were CD70 ${ }^{\text {LOW }}$ (i.e., MBT162). Interestingly, MBT190 had far fewer CD8+ cytotoxic tumor infiltrating 
Iymphocytes than most samples, including MBT162 (SupplFig6A). In the CD3+ Iymphoid population, we found that while CD27 was expressed on the majority of these cells, very few of them expressed CD70 (Fig5A, SupplFig6A), in agreement with existing literature (Sánchez-Martínez et al. 2019). Lastly, we noted that CD27 was highly expressed on CD4 helper $\mathrm{T}$ cells, potentially making them more sensitive to $\mathrm{CD} 70^{\mathrm{HIGH}}$ GBMs. Together, these data indicate that multiple CD27/CD70-axis interactions are occurring within the GBM TIME, likely contributing to the low immunogenicity of rGBM.

\section{DISCUSSION}

Since the creation of the Stupp protocol in 2005, few advances have been made in bringing new therapeutics to market for GBM. In addition, resistance to SoC treatment has begun to direct therapeutic investigation towards a small reservoir of cells termed BTICs (Osuka and Van Meir, 2017). BTICs display enhanced self-renewal properties and are believed to be capable of de novo tumor formation, and driving tumor recurrence (Osuka and Van Meir, 2017). It is believed that SoC therapy helps drive recurrent tumors by creating a bottleneck, with cells that escape initial chemoradiotherapy driving formation of a therapy-resistant recurrent tumor. Thus, recent endeavors have sought to identify actionable targets on this subpopulation of cells. Numerous clinical trials against these targets have assessed the viability of CAR-T cells against different antigens, dendritic cell vaccines and immune checkpoint inhibitors, amongst others. However, despite many efforts little progress has been made, and most recurrent GBM patients are destined for clinical trials or palliative care.

Here, we utilized a multi-omics approach using our in-house collection of lowpassage, patient-derived BTICs, and existing public datasets to identify a GBM cell surface marker of treatment-resistant BTICs. Using this approach, we identified and validated CD70 as a promising therapeutic target in recurrent GBM.

From the literature, CD70 seems to have a confounding role in cancer, both stimulating and suppressing immune response in various cancers (Rowley and AlShamkhani, 2004; Aulwurm et al. 2006). With this in mind, we sought not only to validate CD70 as a therapeutic target in GBM, but also to better understand its role in GBM cell maintenance and progression, as well as the tumor immune microenvironment. We 
demonstrate that CD70 is vital in sphere formation and proliferation of GBM cells, two essential BTIC characteristics which correlate with ability to recapitulate GBM tumors in vivo. To follow, we show that CD70 silencing significantly reduce tumor burden and volume, and significantly increases survival time, with some animals showing no signs of disease up until the end of the experiment. These data represent similar findings to that seen in the literature, indicating that CD70 may play a crucial role in tumor-initiating cells in multiple cancers (Liu et al. 2018; Nakamura et al. 2021). To better understand the cellular pathways and programs involving CD70, we carried out GSEA using RNAseq of our CD70-silenced cell lines. We were able to contribute to the functional understanding of CD70 by showing that, as previously noted in RCC and other cancers, CD70 plays a role in controlling hypoxia (Ruf, Moch and Schrami, 2016; Kitajima et al. 2018). However, this is the first time to our knowledge that the regulatory role of CD70 in hypoxia has been demonstrated in GBM specifically, or brain cancer in general. Hypoxia is the consequence of poor vascularization, and thus poor blood delivery, within the tumor. This often promotes cancer cell spreading via invasion so that cells may escape the low-oxygen environment, thereby rendering the tumor diffuse and far more aggressive, a characteristic often seen in recurrent GBM. Based on our GSEA, it is possible that the vascularization factor VEGF is dependent on CD70 expression and the CD70/CD27 signalling axis, leading to, and tumor neoangiogenesis, a role previously established in non-cancer pathologies (Simons et al. 2018). Our analysis also revealed that CD70 silencing appeared to depress pathways related to EMT signaling, a program that is linked to a more aggressive cancer capable of therapy evasion in multiple cancers, including GBM (Tulchinsky et al. 2019; Zheng et al. 2015; Perotti et al. 2019; Tang et al. 2016).

By defining the tumorigenic significance of CD70 in GBM, we sought to develop potential therapeutic modalities directed against CD70. We developed a CAR against CD70 and demonstrated its efficacy both in vitro and in vivo, where it displayed high specificity for CD70, and conferred significantly extended survival in our orthotopically xenografted animal models, with some animals experiencing complete remission. CD70CAR-T cell efficacy varied slightly in vivo, which may be due to the extensive heterogeneity of GBM, particularly in early passage GBM BTICs which recapitulate intratumoral heterogeneity quite well. Changes in associated clonal dynamics which arise 
with therapeutic pressure may generate therapy-resistant CD70 LOW subpopulations with antigen escape, as has been seen in the clinic (Kim et al. 2015). While antigen escape is a major problem at the moment, particularly as not all BTICs uniformly express CD70 (SupplFig1B) or any other single targetable protein, it is may be possible to overcome this issue using a poly-therapeutic strategy. Examples of such a combinatorial strategy include a bispecific anti-CD70/SIRPa antibody which outperforms individually delivered antibodies in models of human Burkitt's lymphoma, allowing for co-targeting of both tumor cells and tumor-associated macrophages in the TIME (Ring et al. 2017, https://doi.org/10.1073/pnas.1710877114). We also detected a population of CD27+ proinflammatory putative 'M1' macrophages in our profiling of patient-derived rGBM TIME samples, indicating that while the TIME is immunosuppressive, it does contain traditionally anti-tumor components. CD27 is a marker of highly immunosuppressive Tregs (Starzer and Berghoff, 2020), and it is possible that tumor associated macrophages and other members of the TIME may exert some of their immunosuppressive effects through CD27/CD70 interactions, inducing a more immunosuppressive phenotype in tumor infiltrating lymphocytes (Jacobs et al. 2015). Among the TCGA matched pairs, we noticed a trend of subtype switching to the mesenchymal subtype upon recurrence, as has been observed in the literature (Segerman et al. 2016, Liu et al. 2018), and increased CD70 expression correlated with the mesenchymal subtype as well. The mesenchymal subtype is associated with a more aggressive tumor, as well as invasion, therapy evasion and a poorer prognosis (Q. Wang et al. 2017; Pich, C et al. 2016), and has been shown to harbour a stronger immunosuppressive microenvironment. While initially this may seem to be negative as far as the impact cell therapies might have, some studies suggest the opposite, and that immunotherapies may have a more drastic impact on these tumors (Haddad et al. 2020; Chen and Hambardzumyan, 2018).

In accordance with the literature, after silencing of CD70 expression in various GBM cell lines, we found that CD70 repression results in downregulation of IFN- $\alpha$ and IL1 , both of which play an important role in the Th1 lymphocyte response, and are known for their pro-inflammatory role in tumors (Biron, 1998; B. S. Kim et al. 2012). In conjunction with other work highlighting CAR-T cells' ability to induce inflammation (Gajewski et al. 2017), we see the potential for CD70CAR-T cells to convert the GBM microenvironment 
from immunologically "cold" to "hot", eliciting an anti-tumor immune response of endogenous effector cells. It has previously been shown that certain aspects of T cell functionality are dependent on IFN- $\alpha$ and CD70 (Allam et al. 2014), although to our knowledge this is the first time that this dependence has been shown in cancer cells.

Our work, as well as recent work from others (Ge et al. 2017), maintain that CD70 is a promising target for rGBM, and a better understanding of the role of CD70 in the GBM TIME is needed. In particular, we advocate for determining the role that CD70 plays in initiating GBM recurrence and potential mechanisms of therapy evasion. While the literature shows conflicting results regarding whether CD70 plays a pro- or antitumorigenic effect in cancer, we postulate a novel mechanism, through which continued stimulation of the CD70/CD27 axis leads to continuous T cell activation by GBM cells, and subsequent exhaustion within the T-cell compartment, as previously observed in models of HIV (Tesselaar et al. 2003).

As has been investigated in the literature, CD70 is present on the cell surface of T-cells, however, it does not play a functional role, and as a result, other groups have noted fratricide in their CD70 CAR-T cell populations (Wang, Q. J. et al. 2018). Based on these reports, as well as our own observations collected from our CD70 CAR-T cell populations, we developed a model of CD70-CAR T cell fratricide using Jurkat cells, which may be used as a platform for future studies in adoptive cell therapy. Reasons for the observed decrease in viability of Jurkat cells, though not assessed experimentally here, have previously been speculated upon by colleagues using CAR-transduced Jurkat cells (Raikar et al. 2017), reporting that cell death occurs due to prolonged activationinduced paracrine and autocrine interactions. Compared to the flow cytometry-sorted CD70-enriched Jurkat cells used in our experiments, CD70 expression is relatively scarce on activated T cells, indicating that fratricide would occur to a far lesser extent on natural PBMC-derived CD70 CAR-T cells. In our hands, only small variations in cell viability were observed in our CD70 CAR-T cell experiments. Nonetheless, others recently reported an additional fitness benefit in a CD70 knockout CD70 CAR-T cell, which conferred various functional benefits including increased proliferation and cytotoxicity, and was far more advantageous than other obvious knockout targets such as PD-1 and LAG3 (Dequeant et al, 2021). Thus, we highly encourage future studies exploring any potential benefit a 
CD70KO CD70 CAR-T cell may display in GBM, where the TIME is notoriously difficult to overcome. Targeted delivery of the CAR construct directly into the CD70 locus would disrupt CD70 expression, preventing undesirable stimulation and enhancing proliferation and cytotoxicity of CD70 CAR-T cells (Kumar et al. 2020). Strategies involving targeted gene delivery have already been applied to an array of other targets to optimize adoptive cell therapies (Cooper et al. 2018).

Here, we investigated the functional benefit that CD70 expression confers in GBM cells, and the implied influence that CD70 expression may have on interactions with the immunosuppressive landscape. We employed a reverse translational approach (Goswami et al. 2020) to determine CD27's expression pattern - CD70s only known receptor - in different compartments of the GBM TIME. This highlights the influence that CD70-expressing GBMs may have on their microenvironment by leveraging this interaction, and how utilizing a 'double jeopardy' therapeutic strategy - targeting both GBM cells and immunosuppressive microenvironment cells - may result in highly potent anti-tumor activity. Considering our data and that of recent clinical trials targeting CD70 by systemic administration (Riether et al. 2020), we believe intracranially delivered CD70 CAR-T therapy holds great promise, and should be explored alone and in conjunction with TIME-targeting therapeutics. 


\section{Main Figures:}

Figure 1: CD70 expression is a relevant marker of recurrent glioblastoma.

A: Manhattan plot of the top upregulated RNAseq genes in the recurrent patient-derived GBM brain tumor initiating cells (rGBM BTICs) BT241, compared to the publicly available TCGA database, identified CD70 (circled) as a target candidate upregulated in the BTIC subpopulation, compared to tumor bulk. B: Analysis of CD70 mRNA levels in the five TGCA primary/recurrent patient matched GBM pairs depicts an CD70 increase in three pairs upon recurrence, alongside a switch towards the Mesenchymal subtype (GBM subtype classification: $\mathrm{C}$, classical; $\mathrm{P}$, proneural; $\mathrm{M}$, mesenchymal). C: Among GBM BTICs from Figure 1C, the two in-house matched p/rGBM BTICs pairs display an increase of CD70 cell surface expression upon tumor recurrence. D: Box and whiskers representation of a higher CD70 cell surface expression in rGBM BTICs compared to primary (p-) GBM BTICS and normal brain cells (astrocytes, neural stem cells), assessed by flow cytometry (Singh lab brain tumor database). E: Volcano plot of the top up- and down-regulated cell surface proteins of pair 2 from Figure 1D, as assessed by glycocapture proteomics.

Figure 2: CD70 is a dedicated player in GBM maintenance and tumor formation.

A: GBM BTICs were sorted into positive and negative populations and proliferation was assessed by PrestoBlue assay B: Silencing of CD70 expression by shRNA (shCD70) knockdown and sphere formation ability was assessed compared to shGFP (Control shRNA). C - F: Immunocompromised mice (NSG, a minimum of six mice per condition) were intracranially injected with shGFP or shCD70 BTICs. C, D: Tumor area of CD70-silenced BTICs compared to control knockdown BTICs was measured using formalin-fixed, H\&E-stained mouse brain slices (right, representative picture). E,F: Kaplan-Meier survival curves comparing mice engrafted with shCD70 BTICs compared to shGFP BTICs. The two remaining BT241 shCD70 mice at the end of experiment showed an absence of tumor by H\&E staining at experimental endpoint (data not shown). G: MRI images representative of xenografts from shGFP and shCD70 transduced GBM BTIC line BT241. Right panels are control 
images of normal mouse brain. $\left({ }^{*}=p<0.05 ;{ }^{* *}=p<0.01 ;{ }^{* *}=p<0.001 ;{ }^{* * *}=\right.$ $p<0.0001)$

Figure 3: Generation and in vitro Characterization of CD70-Specific CAR-T Cells.

A: Binding curve comparing CD70-specific Fabs to commercial standard antibody. B: Anti-CD70 Fab'2 is specific against CD70, assessed by cytotoxicity assay under combination treatment with $2^{\circ} \mathrm{ADC}$, against GBM cells expressing high (GBM BTIC BT241) or no (HEK293) CD70. C: Schematic representation of CAR structure. D: Successful transduction of CAR-T vectors as observed by NGFR+ cells in ConCAR-T cells and NGFR+Myc+ cells in CD70 CAR-T cells, displayed as a representative flow plot. E: Testing of CAR-T cell activation; IFN-gamma and TNFalpha cytokine released during coculture of GBM BTIC BT241 with CD70 CAR-T, compared to ConCAR-T cells, as analyzed by ELISA assay $(n=3)$. F: Cytotoxicity assay to assess CD70CAR killing capacity compared to ConCAR after co-culturing for 24 hours, tested at various effector to target $(E: T)$ ratios $(n=3) .\left({ }^{*}=p<0.05 ;{ }^{* *}=p<0.01 ;{ }^{* *}\right.$ $\left.=p<0.001 ;{ }^{* * *}=p<0.0001\right)$

Figure 4: CD70 CAR-T are efficacious against recurrent GBM tumors in vivo. NSG mice (at least $n=6$ per group) were intracranially implanted with 100,000 human BT241 ffLuc GBM cells. Upon successful engraftment, mice were treated with $1 \times 10^{6}$ CD70CAR-T or ConCAR-T cells, delivered intracranially once a week for two weeks. A: CD70 CAR-T treated mice showed decreased tumor signal, as assessed by bioluminescence measurement (right, representative picture of radiance measurement in the region of interest). A lower tumor burden was observed in the CD70 CAR-T group compared to the control group, as measured on B: formalin-fixed, H\&E stained mouse brain slices (representative picture on the right), and C: an extended survival (Kaplan-Meier curve) $\left({ }^{*}=p<0.05 ;{ }^{* *}=p<0.01 ;{ }^{* *}=p<0.001 ;{ }^{* * *}=p<0.0001\right.$ )

\section{Figure 5: Modelling of CD70 influence on GBM TIME.}

Tumor immune microenvironment (TIME) cells extracted from patient tumor samples were analyzed by flow cytometry, evaluating the pattern of expression of CD27 in non- 
lymphoid (CD45+CD3-) and M1 populations (CD45+CD3-CD68+HLADR+). A,

B: Average expression of CD27 on CD4/CD8 lymphoid population, and CD70 expression on the lymphoid population (CD3+). C: CD70 expression kinetics on inhouse, activated T cells and D: levels of CD69 and cMyc displayed by CD70CAR or ConCAR-T cells nine days post-transduction, evaluated by flow cytometry. E: CD70enriched or -silenced Jurkat cells were transduced with either ConCAR or CD70CAR. After 8 days CD69 and CD70 levels were assessed by flow cytometry. $\left({ }^{*}=p<0.05\right.$; ${ }^{* *}=$ $\left.p<0.01 ;{ }^{* * *}=p<0.001 ;{ }^{* * *}=p<0.0001\right)$

\section{Supplementary Figures:}

\section{Supp Figure 1: CD70 expression on in-house primary and recurrent GBM samples.}

A. Manhattan plot of the top upregulated RNAseq genes in patient-derived GBM brain tumor initiating cells (GBM BTICs) BT698, BT956, BT618, compared to the publicly available TCGA database, identified CD70 (circled in red) as a target candidate upregulated in the BTIC subpopulation, compared to tumor bulk. B: CD70 cell surface protein expression, assessed by flow cytometry cell surface staining on multiple inhouse primary and recurrent cell lines, as well as normal human cell lines.

\section{Supp Figure 2: Knockdown of CD70 improves survival in vivo.}

A: Proliferation of sorted CD70-positive and -negative cells from BT698, BT428, BT458 GBM cultures was assessed using a PrestoBlue assay. B: Cell surface CD70 expression after shRNA knockdown in three CD70 ${ }^{\mathrm{HIGH}}$ GBM lines, as assessed by flow cytometry. C: Tumor area from animal-engrafted tumors of shCD70 GBM4 cells compared to control. D: Kaplan-Meier curve displaying survival of mice engrafted with shCD70 GBM4 cells compared to shGFP GBM4 controls. 


\section{Supplementary Figure 3: CD70 influence on the MES subtype and associated} machinery.

A, B: RNA sequencing of CD70 silenced GBM BTIC cells BT241, GBM8 and GBM4 permitted the gene set enrichment analysis (GSEA) (A) and Cytoscape Node map (B) depicting circuitries under CD70 dependence.

\section{Supp Figure 4: Generation and in vitro Characterization of CD70-Specific CAR-T} Cells.

A: Internalization studies showing Fab presence on the surface of BT241 cells after a 2 hour incubation. B, C: IFN-gamma and TNF-alpha release during coculture of CD70 CAR-T or ConCAR with GBM BTICs lines GBM4, GBM8, or BT935 CD70 CAR-T, at effector to target (E:T) ratios of 1:1, as analyzed by ELISA ( $n=3)$. D: Cytotoxicity assay assessing the killing capacity of CD70CAR-T cells on GBM8 BTICs compared to control, after co-culturing for 24 hours, as tested at various $E: T$ ratios $(n=3)$.

\section{Supp Figure 5: CD70 CAR-Ts are efficacious against recurrent GBM8 cells in vivo.}

NSG mice (at least $n=6$ per group) were intracranially implanted with 100,000 human GBM8 ffLuc GBM cells. Upon successful engraftment, mice were treated with $1 \times 10^{6}$ CD70CAR-T or ConCAR-T cells, delivered intracranially once a week for two weeks. A: CAR-T treated mice a lower tumor burden in the CD70 CAR-T group compared to Control group as by IVIS imaging. B: CD70CAR-T treated mice also showed higher survival rate compared to that of the ConCAR-T cohort. C: Tumor burden was assessed in the CD70 CAR-T group compared to Control group, as measured using formalin-fixed, H\&E stained mouse brain slices (representative picture on the right).

\section{Supp Figure 6: Modelling CD70s influence on the GBM TIME.}


A. Tumor immune microenvironment (TIME) cells extracted from patient tumor samples were analyzed by flow cytometry, evaluating the pattern of expression of CD27 in nonlymphoid cells (CD45+CD3+) as well as cytotoxic T cell infiltration (CD8+ in

CD45+CD3+). B. Viability of CD3+ or CD3+CD27+ cells were assessed after co-culture with cells only or supernatant only from i) control knockdown BT241 cells; ii) one of two constructs that produce CD70KD BT241 cells.

\section{References:}

Adam, P J, J A Terrett, G Steers, L Stockwin, J A Loader, G C Fletcher, L-S Lu, et al. 2006. "CD70 (TNFSF7) Is Expressed at High Prevalence in Renal Cell Carcinomas and Is Rapidly Internalised on Antibody Binding." British Journal of Cancer 95 (3): 298-306. https://doi.org/10.1038/sj.bjc.6603222.

Aftimos, Philippe, Christian Rolfo, Sylvie Rottey, Fritz Offner, Dominique Bron, Marie Maerevoet, Jean-Charles Soria, et al. 2017. "Phase I Dose-Escalation Study of the Anti-CD70 Antibody ARGX-110 in Advanced Malignancies." Clinical Cancer Research 23 (21): 6411-20. https://doi.org/10.1158/1078-0432.CCR-17-0613.

Allam, Atef, Melissa Swiecki, William Vermi, Jonathan D. Ashwell, and Marco Colonna. 2014. "Dual Function of CD70 in Viral Infection: Modulator of Early Cytokine Responses and Activator of Adaptive Responses." Journal of Immunology (Baltimore, Md.: 1950) 193 (2): 871-78.

https://doi.org/10.4049/jimmunol.1302429.

Aulwurm, Steffen, Jörg Wischhusen, Manuel Friese, Jannie Borst, and Michael Weller. 2006. "Immune Stimulatory Effects of CD70 Override CD70-Mediated Immune Cell Apoptosis in Rodent Glioma Models and Confer Long-Lasting Antiglioma Immunity in Vivo." International Journal of Cancer 118 (7): 1728-35. https://doi.org/10.1002/ijc.21544.

Bao, Shideng, Qiulian Wu, Roger E. McLendon, Yueling Hao, Qing Shi, Anita B. Hjelmeland, Mark W. Dewhirst, Darell D. Bigner, and Jeremy N. Rich. 2006. "Glioma Stem Cells Promote Radioresistance by Preferential Activation of the DNA Damage Response." Nature 444 (7120): 756-60. https://doi.org/10.1038/nature05236.

Bergmann, Natalie, Claire Delbridge, Jens Gempt, Annette Feuchtinger, Axel Walch, Lucas Schirmer, Wolfram Bunk, Thomas Aschenbrenner, Friederike LiescheStarnecker, and Jürgen Schlegel. 2020. "The Intratumoral Heterogeneity Reflects the Intertumoral Subtypes of Glioblastoma Multiforme: A Regional Immunohistochemistry Analysis." Frontiers in Oncology 10 (April). https://doi.org/10.3389/fonc.2020.00494.

Bielamowicz, Kevin, Shumaila Khawja, and Nabil Ahmed. 2013. "Adoptive Cell Therapies for Glioblastoma." Frontiers in Oncology 3 (November). https://doi.org/10.3389/fonc.2013.00275. 
Biron, C. A. 1998. "Role of Early Cytokines, Including Alpha and Beta Interferons (IFNAlpha/Beta), in Innate and Adaptive Immune Responses to Viral Infections."

Seminars in Immunology 10 (5): 383-90. https://doi.org/10.1006/smim.1998.0138.

Borst, Jannie, Jenny Hendriks, and Yanling Xiao. 2005. "CD27 and CD70 in T Cell and B Cell Activation." Current Opinion in Immunology 17 (3): 275-81. https://doi.org/10.1016/j.coi.2005.04.004.

Bouchart, Christelle, Anne-Laure Trépant, Matthieu Hein, Dirk Van Gestel, and Pieter Demetter. 2020. "Prognostic Impact of Glioblastoma Stem Cell Markers OLIG2 and CCND2." Cancer Medicine 9 (3): 1069-78. https://doi.org/10.1002/cam4.2592.

Bowman, Robert L., Qianghu Wang, Angel Carro, Roel G.W. Verhaak, and Massimo Squatrito. 2017. "GlioVis Data Portal for Visualization and Analysis of Brain Tumor Expression Datasets." Neuro-Oncology 19 (1): 139-41. https://doi.org/10.1093/neuonc/now247.

Brennan, Cameron W., Roel G. W. Verhaak, Aaron McKenna, Benito Campos, Houtan Noushmehr, Sofie R. Salama, Siyuan Zheng, et al. 2013. "The Somatic Genomic Landscape of Glioblastoma." Cell 155 (2): 462-77. https://doi.org/10.1016/j.cell.2013.09.034.

Bristol-Myers Squibb. 2013. "A Phase I, Multicenter, Open-Label, Dose-Escalation, Multidose Study of MDX-1203 in Subjects With Advanced/Recurrent Clear Cell Renal Cell Carcinoma or Relapsed/Refractory B-Cell Non Hodgkin's Lymphoma." Clinical trial registration NCT00944905. clinicaltrials.gov. https://clinicaltrials.gov/ct2/show/NCT00944905.

Cancer Genome Atlas Research Network. 2008. "Comprehensive Genomic Characterization Defines Human Glioblastoma Genes and Core Pathways." Nature 455 (7216): 1061-68. https://doi.org/10.1038/nature07385.

Chahlavi, Ali, Patricia Rayman, Amy L. Richmond, Kaushik Biswas, Renliang Zhang, Michael Vogelbaum, Charles Tannenbaum, Gene Barnett, and James H. Finke. 2005. "Glioblastomas Induce T-Lymphocyte Death by Two Distinct Pathways Involving Gangliosides and CD70." Cancer Research 65 (12): 5428-38. https://doi.org/10.1158/0008-5472.CAN-04-4395.

Chen, Zhihong, and Dolores Hambardzumyan. 2018. "Immune Microenvironment in Glioblastoma Subtypes." Frontiers in Immunology 9. https://doi.org/10.3389/fimmu.2018.01004.

Claus, Christina, Carsten Riether, Christian Schürch, Matthias S. Matter, Tamara Hilmenyuk, and Adrian F. Ochsenbein. 2012. "CD27 Signaling Increases the Frequency of Regulatory T Cells and Promotes Tumor Growth." Cancer Research 72 (14): 3664-76. https://doi.org/10.1158/0008-5472.CAN-11-2791.

Cooper, Matthew L., Jaebok Choi, Karl Staser, Julie K. Ritchey, Jessica M. Devenport, Kayla Eckardt, Michael P. Rettig, et al. 2018. "An 'off-the-Shelf' FratricideResistant CAR-T for the Treatment of T Cell Hematologic Malignancies." Leukemia 32 (9): 1970-83. https://doi.org/10.1038/s41375-018-0065-5.

Cusulin, Carlo, Charles Chesnelong, Pinaki Bose, Misha Bilenky, Karen Kopciuk, Jennifer A. Chan, J. Gregory Cairncross, et al. 2015. "Precursor States of Brain Tumor Initiating Cell Lines Are Predictive of Survival in Xenografts and 
Associated with Glioblastoma Subtypes." Stem Cell Reports 5 (1): 1-9. https://doi.org/10.1016/j.stemcr.2015.05.010.

D’Alessio, Alessio, Gabriella Proietti, Gigliola Sica, and Bianca Maria Scicchitano. 2019.

"Pathological and Molecular Features of Glioblastoma and Its Peritumoral

Tissue." Cancers 11 (4): 469. https://doi.org/10.3390/cancers11040469.

Denoeud, Julie, and Muriel Moser. 2011. "Role of CD27/CD70 Pathway of Activation in Immunity and Tolerance." Journal of Leukocyte Biology 89 (2): 195-203. https://doi.org/10.1189/jlb.0610351.

Dequeant, Mary-Lee, Jason Sagert, Demetri Kalaitzidis, Hui Yu, Ashley Porras, McEwan Brigid, Padalia Zinkal, et al. n.d. "CD70 Knockout: A Novel Approach to Augment CAR-T Cell Function." American Association for Cancer Research.

Diegmann, Julia, Kerstin Junker, Bernhard Gerstmayer, Andreas Bosio, Winfried

Hindermann, Julia Rosenhahn, and Ferdinand von Eggeling. 2005. "Identification of CD70 as a Diagnostic Biomarker for Clear Cell Renal Cell Carcinoma by Gene Expression Profiling, Real-Time RT-PCR and Immunohistochemistry." European Journal of Cancer (Oxford, England: 1990) 41 (12): 1794-1801.

https://doi.org/10.1016/j.ejca.2005.05.005.

Diegmann, Julia, Kerstin Junker, Ivan F. Loncarevic, Susanne Michel, Bettina Schimmel, and Ferdinand von Eggeling. 2006. "Immune Escape for Renal Cell Carcinoma: CD70 Mediates Apoptosis in Lymphocytes." Neoplasia (New York, N.Y.) 8 (11): 933-38. https://doi.org/10.1593/neo.06451.

Feldker, Nora, Fulvia Ferrazzi, Harald Schuhwerk, Sebastian A. Widholz, Kerstin Guenther, Isabell Frisch, Kathrin Jakob, et al. 2020. "Genome-Wide Cooperation of EMT Transcription Factor ZEB1 with YAP and AP-1 in Breast Cancer." The EMBO Journal 39 (17): e103209. https://doi.org/10.15252/embj.2019103209.

Fiscon, Giulia, Federica Conte, and Paola Paci. 2018. "SWIM Tool Application to Expression Data of Glioblastoma Stem-like Cell Lines, Corresponding Primary Tumors and Conventional Glioma Cell Lines." BMC Bioinformatics 19 (Suppl 15). https://doi.org/10.1186/s12859-018-2421-x.

Gajewski, Thomas F., Leticia Corrales, Jason Williams, Brendan Horton, Ayelet Sivan, and Stefani Spranger. 2017. "Cancer Immunotherapy Targets Based on Understanding the T Cell-Inflamed Versus Non-T Cell-Inflamed Tumor Microenvironment." Advances in Experimental Medicine and Biology 1036: 19_ 31. https://doi.org/10.1007/978-3-319-67577-0_2.

Ge, Haitao, Luyan Mu, Linchun Jin, Changlin Yang, Yifan (Emily) Chang, Yu Long, Gabriel DeLeon, et al. 2017a. "Tumor Associated CD70 Expression Is Involved in Promoting Tumor Migration and Macrophage Infiltration in GBM." International Journal of Cancer 141 (7): 1434-44. https://doi.org/10.1002/ijc.30830.

Ge, Haitao, Luyan Mu, Linchun Jin, Changlin Yang, Yifan Emily Chang, Yu Long, Gabriel DeLeon, et al. 2017b. "Tumor Associated CD70 Expression Is Involved in Promoting Tumor Migration and Macrophage Infiltration in GBM." International Journal of Cancer 141 (7): 1434-44. https://doi.org/10.1002/ijc.30830.

Giavridis, Theodoros, Sjoukje J. C. van der Stegen, Justin Eyquem, Mohamad Hamieh, Alessandra Piersigilli, and Michel Sadelain. 2018. "CAR T Cell-Induced Cytokine Release Syndrome Is Mediated by Macrophages and Abated by IL-1 Blockade." Nature Medicine 24 (6): 731-38. https://doi.org/10.1038/s41591-018-0041-7. 
Gilder, Andrew S., Letizia Natali, Danielle M. Van Dyk, Cristina Zalfa, Michael A. Banki, Donald P. Pizzo, Huawei Wang, Richard L. Klemke, Elisabetta Mantuano, and Steven L. Gonias. 2018. "The Urokinase Receptor Induces a Mesenchymal Gene Expression Signature in Glioblastoma Cells and Promotes Tumor Cell Survival in Neurospheres." Scientific Reports 8 (1): 2982. https://doi.org/10.1038/s41598018-21358-1.

Goswami, Sangeeta, Thomas Walle, Andrew E. Cornish, Sreyashi Basu, Swetha Anandhan, Irina Fernandez, Luis Vence, et al. 2020. "Immune Profiling of Human Tumors Identifies CD73 as a Combinatorial Target in Glioblastoma." Nature Medicine 26 (1): 39-46. https://doi.org/10.1038/s41591-019-0694-x.

Grewal, Iqbal S. 2008. "CD70 as a Therapeutic Target in Human Malignancies." Expert Opinion on Therapeutic Targets 12 (3): 341-51. https://doi.org/10.1517/14728222.12.3.341.

Guarda, Greta, Marion Braun, Francesco Staehli, Aubry Tardivel, Chantal Mattmann, Irmgard Förster, Matthias Farlik, et al. 2011. "Type I Interferon Inhibits Interleukin-1 Production and Inflammasome Activation.” Immunity 34 (2): 213-23. https://doi.org/10.1016/j.immuni.2011.02.006.

Haddad, Alexander F., Jia-Shu Chen, Taemin Oh, Matheus P. Pereira, Rushikesh S. Joshi, and Manish K. Aghi. 2020. "Higher Cytolytic Score Correlates with an Immunosuppressive Tumor Microenvironment and Reduced Survival in Glioblastoma." Scientific Reports 10 (1): 17580. https://doi.org/10.1038/s41598020-73793-8.

Hegi, Monika E., Annie-Claire Diserens, Thierry Gorlia, Marie-France Hamou, Nicolas de Tribolet, Michael Weller, Johan M. Kros, et al. 2005. "MGMT Gene Silencing and Benefit from Temozolomide in Glioblastoma." The New England Journal of Medicine 352 (10): 997-1003. https://doi.org/10.1056/NEJMoa043331.

Held-Feindt, Janka, and Rolf Mentlein. 2002. "CD70/CD27 Ligand, a Member of the TNF Family, Is Expressed in Human Brain Tumors." International Journal of Cancer 98 (3): 352-56. https://doi.org/10.1002/ijc.10207.

Hintzen, Rogier Q., Susanne M. A. Lens, Gerrit Koopman, Steven T. Pals, Hergen Spits, and René A. W. van Lier. 1994. "CD70 Represents the Human Ligand for CD27." International Immunology 6 (3): 477-80. https://doi.org/10.1093/intimm/6.3.477.

Hirschhaeuser, Franziska, Heike Menne, Claudia Dittfeld, Jonathan West, Wolfgang Mueller-Klieser, and Leoni A. Kunz-Schughart. 2010. "Multicellular Tumor Spheroids: An Underestimated Tool Is Catching up Again.” Journal of Biotechnology, Organotypic Tissue Culture for Substance Testing, 148 (1): 3-15. https://doi.org/10.1016/j.jbiotec.2010.01.012.

Hishima, T., M. Fukayama, Y. Hayashi, T. Fujii, T. Ooba, N. Funata, and M. Koike. 2000. "CD70 Expression in Thymic Carcinoma." The American Journal of Surgical Pathology 24 (5): 742-46. https://doi.org/10.1097/00000478200005000-00014.

Inaguma, Shingo, Jerzy Lasota, Piotr Czapiewski, Renata Langfort, Janusz Rys, Joanna Szpor, Piotr Waloszczyk, et al. 2020. "CD70 Expression Correlates with a Worse Prognosis in Malignant Pleural Mesothelioma Patients via Immune 
Evasion and Enhanced Invasiveness." The Journal of Pathology 250 (2): 205-16. https://doi.org/10.1002/path.5361.

Jacobs, Julie, Karen Zwaenepoel, Christian Rolfo, Jolien Van den Bossche, Christophe Deben, Karen Silence, Christophe Hermans, et al. 2015. "Unlocking the Potential of CD70 as a Novel Immunotherapeutic Target for Non-Small Cell Lung Cancer." Oncotarget 6 (15): 13462-75. https://doi.org/10.18632/oncotarget.3880.

Jin, Linchun, Haitao Ge, Yu Long, Changlin Yang, Yifan Emily Chang, Luyan Mu, Elias J. Sayour, et al. 2018. "CD70, a Novel Target of CAR T-Cell Therapy for Gliomas.” Neuro-Oncology 20 (1): 55-65. https://doi.org/10.1093/neuonc/nox116. Johnson, Brett E., Tali Mazor, Chibo Hong, Michael Barnes, Koki Aihara, Cory Y. McLean, Shaun D. Fouse, et al. 2014. "Mutational Analysis Reveals the Origin and Therapy-Driven Evolution of Recurrent Glioma." Science 343 (6167): 18993. https://doi.org/10.1126/science.1239947.

Kashima, Jumpei, Yusuke Okuma, Yukio Hosomi, and Tsunekazu Hishima. 2019. "High Serum Soluble CD27 Level Correlates with Poor Performance Status and Reduced Survival in Patients with Advanced Lung Cancer." Oncology 97 (6): 365-72. https://doi.org/10.1159/000502441.

Kim, Byung S., Young-Hee Jin, Liping Meng, Wanqiu Hou, Hyun Seok Kang, Hey Suk Park, and Chang-Sung Koh. 2012. "IL-1 Signal Affects Both Protection and Pathogenesis of Virus-Induced Chronic CNS Demyelinating Disease." Journal of Neuroinflammation 9 (1): 217. https://doi.org/10.1186/1742-2094-9-217.

Kim, Jinkuk, In-Hee Lee, Hee Jin Cho, Chul-Kee Park, Yang-Soon Jung, Yanghee Kim, So Hee Nam, et al. 2015. "Spatiotemporal Evolution of the Primary Glioblastoma Genome." Cancer Cell 28 (3): 318-28. https://doi.org/10.1016/j.ccell.2015.07.013.

Kitajima, Shojiro, Kian Leong Lee, Masaki Fujioka, Wendi Sun, Jia You, Grace Sushin Chia, Hideki Wanibuchi, et al. 2018. "Hypoxia-Inducible Factor-2 Alpha upRegulates CD70 under Hypoxia and Enhances Anchorage-Independent Growth and Aggressiveness in Cancer Cells." Oncotarget 9 (27): 19123-35. https://doi.org/10.18632/oncotarget.24919.

Kumar, Sandeep, Sunil Kumar Singh, Navin Viswakarma, Gautam Sondarva, Rakesh Sathish Nair, Periannan Sethupathi, Matthew Dorman, et al. 2020. "Rationalized Inhibition of Mixed Lineage Kinase 3 and CD70 Enhances Life Span and Antitumor Efficacy of CD8+ T Cells." Journal for Immunotherapy of Cancer 8 (2). https://doi.org/10.1136/jitc-2019-000494.

Lens, S M, P A Baars, B Hooibrink, M H van Oers, and R A van Lier. 1997. "AntigenPresenting Cell-Derived Signals Determine Expression Levels of CD70 on Primed T Cells." Immunology 90 (1): 38-45.

Lens, S. M., P. Drillenburg, B. F. den Drijver, G. van Schijndel, S. T. Pals, R. A. van Lier, and M. H. van Oers. 1999. "Aberrant Expression and Reverse Signalling of CD70 on Malignant B Cells.” British Journal of Haematology 106 (2): 491-503. https://doi.org/10.1046/j.1365-2141.1999.01573.x.

Liberzon, A., A. Subramanian, R. Pinchback, H. Thorvaldsdottir, P. Tamayo, and J. P. Mesirov. 2011. "Molecular Signatures Database (MSigDB) 3.0." Bioinformatics 27 (12): 1739-40. https://doi.org/10.1093/bioinformatics/btr260. 
Linde, Myra E. van, Cyrillo G. Brahm, Philip C. de Witt Hamer, Jaap C. Reijneveld, Anna M. E. Bruynzeel, W. Peter Vandertop, Peter M. van de Ven, et al. 2017. "Treatment Outcome of Patients with Recurrent Glioblastoma Multiforme: A Retrospective Multicenter Analysis." Journal of Neuro-Oncology 135 (1): 183-92. https://doi.org/10.1007/s11060-017-2564-z.

Liu, Gentao, Xiangpeng Yuan, Zhaohui Zeng, Patrizia Tunici, Hiushan Ng, Iman R. Abdulkadir, Lizhi Lu, Dwain Irvin, Keith L. Black, and John S. Yu. 2006. "Analysis of Gene Expression and Chemoresistance of CD133+ Cancer Stem Cells in Glioblastoma." Molecular Cancer 5 (1): 67. https://doi.org/10.1186/1476-4598-567.

Lu, Fanghui, Ying Chen, Chuntao Zhao, Haibo Wang, Danyang He, Lingli Xu, Jincheng Wang, et al. 2016. "Olig2-Dependent Reciprocal Shift in PDGF and EGF Receptor Signaling Regulates Tumor Phenotype and Mitotic Growth in Malignant Glioma." Cancer Cell 29 (5): 669-83. https://doi.org/10.1016/j.ccell.2016.03.027.

Ma, Junliang, Lunxu Liu, Guowei Che, Nanbin Yu, Fuqiang Dai, and Zongbing You. 2010. "The M1 Form of Tumor-Associated Macrophages in Non-Small Cell Lung Cancer Is Positively Associated with Survival Time." BMC Cancer 10 (1): 112. https://doi.org/10.1186/1471-2407-10-112.

McAbee, Joseph H., Charlotte Degorre-Kerbaul, Kristin Valdez, Astrid Wendler, Uma T. Shankavaram, Colin Watts, Kevin Camphausen, and Philip J. Tofilon. 2020. "Detection of Glioblastoma Intratumor Heterogeneity in Radiosensitivity Using Patient-Derived Neurosphere Cultures." Journal of Neuro-Oncology 149 (3): 383-90. https://doi.org/10.1007/s11060-020-03643-0.

McDonagh, Charlotte F., Kristine M. Kim, Eileen Turcott, Lindsay L. Brown, Lori Westendorf, Tiffany Feist, Django Sussman, et al. 2008. "Engineered Anti-CD70 Antibody-Drug Conjugate with Increased Therapeutic Index.” Molecular Cancer Therapeutics 7 (9): 2913-23. https://doi.org/10.1158/1535-7163.MCT-08-0295.

Merico, Daniele, Ruth Isserlin, Oliver Stueker, Andrew Emili, and Gary D. Bader. 2010. "Enrichment Map: A Network-Based Method for Gene-Set Enrichment Visualization and Interpretation." PLOS ONE 5 (11). https://doi.org/10.1371/journal.pone.0013984.

Meyer, Mona, Jüri Reimand, Xiaoyang Lan, Renee Head, Xueming Zhu, Michelle Kushida, Jane Bayani, et al. 2015. "Single Cell-Derived Clonal Analysis of Human Glioblastoma Links Functional and Genomic Heterogeneity." Proceedings of the National Academy of Sciences 112 (3): 851-56. https://doi.org/10.1073/pnas.1320611111.

Morgan, Richard A., Laura A. Johnson, Jeremy L. Davis, Zhili Zheng, Kevin D. Woolard, Elizabeth A. Reap, Steven A. Feldman, et al. 2012. "Recognition of Glioma Stem Cells by Genetically Modified T Cells Targeting EGFRvIII and Development of Adoptive Cell Therapy for Glioma." Human Gene Therapy 23 (10): 1043-53. https://doi.org/10.1089/hum.2012.041.

Munitic, Ivana, Mirela Kuka, Atef Allam, Jonathan P. Scoville, and Jonathan D. Ashwell. 2013. "CD70-Deficiency Impairs Effector CD8 T Cell Generation and Viral Clearance but Is Dispensable for the Recall Response to LCMV." Journal of Immunology (Baltimore, Md. : 1950) 190 (3): 1169-79. https://doi.org/10.4049/jimmunol.1202353. 
Nakamura, Kota, Masayuki Sho, Takahiro Akahori, Satoshi Nishiwada, Tomohiro Kunishige, Kenji Nakagawa, Minako Nagai, Tadataka Takagi, Taichi Terai, and Naoya Ikeda. 2021. "Clinical Relevance of CD70 Expression in Resected Pancreatic Cancer: Prognostic Value and Therapeutic Potential." Pancreatology: Official Journal of the International Association of Pancreatology (IAP) ... [et AI.] 21 (3): 573-80. https://doi.org/10.1016/j.pan.2021.01.013.

Neftel, Cyril, Julie Laffy, Mariella G. Filbin, Toshiro Hara, Marni E. Shore, Gilbert J. Rahme, Alyssa R. Richman, et al. 2019. "An Integrative Model of Cellular States, Plasticity and Genetics for Glioblastoma." Cell 178 (4): 835-849.e21. https://doi.org/10.1016/j.cell.2019.06.024.

Nilsson, Anna, Angelo de Milito, Frida Mowafi, Gösta Winberg, Olle Björk, Elisabeth Z. Wolpert, and Francesca Chiodi. 2005. "Expression of CD27-CD70 on Early B Cell Progenitors in the Bone Marrow: Implication for Diagnosis and Therapy of Childhood ALL." Experimental Hematology 33 (12): 1500-1507. https://doi.org/10.1016/j.exphem.2005.10.005.

Nolte, Martijn A., Ronald W. van Olffen, Klaas P. J. M. van Gisbergen, and René A. W. van Lier. 2009. "Timing and Tuning of CD27-CD70 Interactions: The Impact of Signal Strength in Setting the Balance between Adaptive Responses and Immunopathology." Immunological Reviews 229 (1): 216-31. https://doi.org/10.1111/j.1600-065X.2009.00774.x.

Ostrom, Quinn T., Gino Cioffi, Haley Gittleman, Nirav Patil, Kristin Waite, Carol Kruchko, and Jill S. Barnholtz-Sloan. 2019. "CBTRUS Statistical Report: Primary Brain and Other Central Nervous System Tumors Diagnosed in the United States in 2012-2016." Neuro-Oncology 21 (Suppl 5): v1-100. https://doi.org/10.1093/neuonc/noz150.

Osuka, Satoru, and Erwin G. Van Meir. 2017. "Overcoming Therapeutic Resistance in Glioblastoma: The Way Forward." The Journal of Clinical Investigation 127 (2): 415-26. https://doi.org/10.1172/JCI89587.

Pahl, Jens Hw, Susy J. Santos, Marieke L. Kuijjer, Gerharda H. Boerman, Laurens GI Sand, Karoly Szuhai, Annemarie Cleton-Jansen, et al. 2015. "Expression of the Immune Regulation Antigen CD70 in Osteosarcoma." Cancer Cell International 15: 31. https://doi.org/10.1186/s12935-015-0181-5.

Patel, Anoop P., Itay Tirosh, John J. Trombetta, Alex K. Shalek, Shawn M. Gillespie, Hiroaki Wakimoto, Daniel P. Cahill, et al. 2014. "Single-Cell RNA-Seq Highlights Intratumoral Heterogeneity in Primary Glioblastoma." Science 344 (6190): 13961401. https://doi.org/10.1126/science.1254257.

Perotti, Valentina, Paola Baldassari, Alessandra Molla, Gabriella Nicolini, Ilaria Bersani, Giulia Grazia, Fabio Benigni, et al. 2019. "An Actionable Axis Linking NFATc2 to EZH2 Controls the EMT-like Program of Melanoma Cells." Oncogene 38 (22): 4384-96. https://doi.org/10.1038/s41388-019-0729-2.

Pich, Christine, Guillaume Sarrabayrouse, lotefa Teiti, Bernard Mariamé, Philippe Rochaix, Laurence Lamant, Gilles Favre, Véronique Maisongrosse, and AnneFrançoise Tilkin-Mariamé. 2016. "Melanoma-Expressed CD70 Is Involved in Invasion and Metastasis." British Journal of Cancer 114 (1): 63-70. https://doi.org/10.1038/bjc.2015.412. 
Priceman, Saul J., Stephen J. Forman, and Christine E. Brown. 2015. "Smart CARs Engineered for Cancer Immunotherapy." Current Opinion in Oncology 27 (6): 466-74. https://doi.org/10.1097/CCO.0000000000000232.

Purdue, Mark P., Qing Lan, Judith Hoffman-Bolton, Allan Hildesheim, Catherine L. Callahan, Paul Strickland, Kala Visvanathan, and Nathaniel Rothman. 2019. "Circulating SCD27 and SCD30 in Pre-Diagnostic Samples Collected Fifteen Years Apart and Future Non-Hodgkin Lymphoma Risk." International Journal of Cancer 144 (8): 1780-85. https://doi.org/10.1002/ijc.31879.

Rahman, Maryam, Jesse Kresak, Changlin Yang, Jianping Huang, Wesley Hiser, Paul Kubilis, and Duane Mitchell. 2018. "Analysis of Immunobiologic Markers in Primary and Recurrent Glioblastoma." Journal of Neuro-Oncology 137 (2): 249_ 57. https://doi.org/10.1007/s11060-017-2732-1.

Raikar, Sunil S., Lauren C. Fleischer, Robert Moot, Andrew Fedanov, Na Yoon Paik, Kristopher A. Knight, Christopher B. Doering, and H. Trent Spencer. 2017. "Development of Chimeric Antigen Receptors Targeting T-Cell Malignancies Using Two Structurally Different Anti-CD5 Antigen Binding Domains in NK and CRISPR-Edited T Cell Lines." Oncoimmunology 7 (3). https://doi.org/10.1080/2162402X.2017.1407898.

Riether, Carsten, Thomas Pabst, Sabine Höpner, Ulrike Bacher, Magdalena Hinterbrandner, Yara Banz, Rouven Müller, et al. 2020. "Targeting CD70 with Cusatuzumab Eliminates Acute Myeloid Leukemia Stem Cells in Patients Treated with Hypomethylating Agents." Nature Medicine 26 (9): 1459-67. https://doi.org/10.1038/s41591-020-0910-8.

Ring, Nan Guo, Dietmar Herndler-Brandstetter, Kipp Weiskopf, Liang Shan, Jens-Peter Volkmer, Benson M. George, Melanie Lietzenmayer, et al. 2017. "Anti-SIRPa Antibody Immunotherapy Enhances Neutrophil and Macrophage Antitumor Activity." Proceedings of the National Academy of Sciences of the United States of America 114 (49): E10578-85. https://doi.org/10.1073/pnas.1710877114.

Robin, Adam M., Ian Lee, and Steven N. Kalkanis. 2017. "Reoperation for Recurrent Glioblastoma Multiforme." Neurosurgery Clinics of North America 28 (3): 407-28. https://doi.org/10.1016/j.nec.2017.02.007.

Rønning, Pål A., Eirik Helseth, Torstein R. Meling, and Tom B. Johannesen. 2012. "A Population-Based Study on the Effect of Temozolomide in the Treatment of Glioblastoma Multiforme." Neuro-Oncology 14 (9): 1178-84. https://doi.org/10.1093/neuonc/nos153.

Rowley, Tania F., and Aymen Al-Shamkhani. 2004. "Stimulation by Soluble CD70 Promotes Strong Primary and Secondary CD8+ Cytotoxic T Cell Responses in Vivo." Journal of Immunology (Baltimore, Md.: 1950) 172 (10): 6039-46. https://doi.org/10.4049/jimmunol.172.10.6039.

Ruf, Melanie, Holger Moch, and Peter Schraml. 2015. "Interaction of Tumor Cells with Infiltrating Lymphocytes via CD70 and CD27 in Clear Cell Renal Cell Carcinoma." Oncoimmunology 4 (12): e1049805. https://doi.org/10.1080/2162402X.2015.1049805.

Sa, Jason K., Nakho Chang, Hye Won Lee, Hee Jin Cho, Michele Ceccarelli, Luigi Cerulo, Jinlong Yin, et al. 2020. "Transcriptional Regulatory Networks of Tumor- 
Associated Macrophages That Drive Malignancy in Mesenchymal Glioblastoma." Genome Biology 21 (1): 216. https://doi.org/10.1186/s13059-020-02140-x.

Sánchez-Martínez, Diego, Matteo L. Baroni, Francisco Gutierrez-Agüera, Heleia RocaHo, Oscar Blanch-Lombarte, Sara González-García, Montserrat Torrebadell, et al. 2019. "Fratricide-Resistant CD1a-Specific CAR T Cells for the Treatment of Cortical T-Cell Acute Lymphoblastic Leukemia." Blood 133 (21): 2291-2304. https://doi.org/10.1182/blood-2018-10-882944.

Schäfer, Niklas, Gerrit H. Gielen, Laurèl Rauschenbach, Sied Kebir, Andreas Till, Roman Reinartz, Matthias Simon, et al. 2019. "Longitudinal Heterogeneity in Glioblastoma: Moving Targets in Recurrent versus Primary Tumors." Journal of Translational Medicine 17 (1): 96. https://doi.org/10.1186/s12967-019-1846-y.

Seagen Inc. 2018. "A Phase 1 Trial of SGN-CD70A in Patients With CD70-Positive Malignancies." Clinical trial registration NCT02216890. clinicaltrials.gov. https://clinicaltrials.gov/ct2/show/NCT02216890.

Segerman, Anna, Mia Niklasson, Caroline Haglund, Tobias Bergström, Malin Jarvius, Yuan Xie, Ann Westermark, et al. 2016. "Clonal Variation in Drug and Radiation Response among Glioma-Initiating Cells Is Linked to Proneural-Mesenchymal Transition." Cell Reports 17 (11): 2994-3009. https://doi.org/10.1016/j.celrep.2016.11.056.

Shaffer, Donald R., Barbara Savoldo, Zhongzhen Yi, Kevin K. H. Chow, Sunitha Kakarla, David M. Spencer, Gianpietro Dotti, et al. 2011. "T Cells Redirected against CD70 for the Immunotherapy of CD70-Positive Malignancies." Blood 117 (16): 4304-14. https://doi.org/10.1182/blood-2010-04-278218.

Simons, K. H., Z. Aref, H. a. B. Peters, S. P. Welten, A. Y. Nossent, J. W. Jukema, J. F. Hamming, R. Arens, M. R. de Vries, and P. H. A. Quax. 2018. "The Role of CD27-CD70-Mediated T Cell Co-Stimulation in Vasculogenesis, Arteriogenesis and Angiogenesis." International Journal of Cardiology 260 (June): 184-90. https://doi.org/10.1016/j.ijcard.2018.02.015.

Singh, Sheila K., Cynthia Hawkins, Ian D. Clarke, Jeremy A. Squire, Jane Bayani, Takuichiro Hide, R. Mark Henkelman, Michael D. Cusimano, and Peter B. Dirks. 2004. "Identification of Human Brain Tumour Initiating Cells." Nature 432 (7015): 396-401. https://doi.org/10.1038/nature03128.

Skaga, Erlend, Evgeny Kulesskiy, Artem Fayzullin, Cecilie J. Sandberg, Swapnil Potdar, Aija Kyttälä, Iver A. Langmoen, et al. 2019. "Intertumoral Heterogeneity in Patient-Specific Drug Sensitivities in Treatment-Naïve Glioblastoma." BMC Cancer 19 (1): 628. https://doi.org/10.1186/s12885-019-5861-4.

Soeda, Akio, Akira Hara, Takahiro Kunisada, Shin-ichi Yoshimura, Toru Iwama, and Deric M. Park. 2015. "The Evidence of Glioblastoma Heterogeneity." Scientific Reports 5 (1): 7979. https://doi.org/10.1038/srep07979.

Starzer, Angelika M., and Anna S. Berghoff. 2020. "New Emerging Targets in Cancer Immunotherapy: CD27 (TNFRSF7)." ESMO Open 4 (Suppl 3): e000629. https://doi.org/10.1136/esmoopen-2019-000629.

Stupp, Roger, Warren P. Mason, Martin J. van den Bent, Michael Weller, Barbara Fisher, Martin J. B. Taphoorn, Karl Belanger, et al. 2005. "Radiotherapy plus Concomitant and Adjuvant Temozolomide for Glioblastoma." The New England Journal of Medicine 352 (10): 987-96. https://doi.org/10.1056/NEJMoa043330. 
Stupp, Roger, Sophie Taillibert, Andrew Kanner, William Read, David Steinberg, Benoit Lhermitte, Steven Toms, et al. 2017. "Effect of Tumor-Treating Fields Plus Maintenance Temozolomide vs Maintenance Temozolomide Alone on Survival in Patients With Glioblastoma: A Randomized Clinical Trial.” JAMA 318 (23): 230616. https://doi.org/10.1001/jama.2017.18718.

Subramanian, Aravind, Pablo Tamayo, Vamsi K. Mootha, Sayan Mukherjee, Benjamin L. Ebert, Michael A. Gillette, Amanda Paulovich, et al. 2005. "Gene Set Enrichment Analysis: A Knowledge-Based Approach for Interpreting GenomeWide Expression Profiles." Proceedings of the National Academy of Sciences 102 (43): 15545-50. https://doi.org/10.1073/pnas.0506580102.

Tang, Haitao, Jiaxin Zhao, Liangyu Zhang, Jiang Zhao, Yongzhi Zhuang, and Peng Liang. 2016. "SRPX2 Enhances the Epithelial-Mesenchymal Transition and Temozolomide Resistance in Glioblastoma Cells." Cellular and Molecular Neurobiology 36 (7): 1067-76. https://doi.org/10.1007/s10571-015-0300-9.

Tao, Chuming, Kai Huang, Jin Shi, Qing Hu, Kuangxun Li, and Xingen Zhu. 2020. "Genomics and Prognosis Analysis of Epithelial-Mesenchymal Transition in Glioma." Frontiers in Oncology 10. https://doi.org/10.3389/fonc.2020.00183.

Tesselaar, Kiki, Ramon Arens, Gijs M. W. van Schijndel, Paul A. Baars, Martin A. van der Valk, Jannie Borst, Marinus H. J. van Oers, and René A. W. van Lier. 2003. "Lethal T Cell Immunodeficiency Induced by Chronic Costimulation via CD27CD70 Interactions." Nature Immunology 4 (1): 49-54. https://doi.org/10.1038/ni869.

Tulchinsky, Eugene, Oleg Demidov, Marina Kriajevska, Nickolai A. Barlev, and Evgeny Imyanitov. 2019. "EMT: A Mechanism for Escape from EGFR-Targeted Therapy in Lung Cancer." Biochimica et Biophysica Acta (BBA) - Reviews on Cancer 1871 (1): 29-39. https://doi.org/10.1016/j.bbcan.2018.10.003.

Venugopal, Chitra, Nicole M. McFarlane, Sara Nolte, Branavan Manoranjan, and Sheila K. Singh. 2012. "Processing of Primary Brain Tumor Tissue for Stem Cell Assays and Flow Sorting." Journal of Visualized Experiments: JoVE, no. 67 (September). https://doi.org/10.3791/4111.

Verhaak, Roel G. W., Katherine A. Hoadley, Elizabeth Purdom, Victoria Wang, Yuan Qi, Matthew D. Wilkerson, C. Ryan Miller, et al. 2010. "Integrated Genomic Analysis Identifies Clinically Relevant Subtypes of Glioblastoma Characterized by Abnormalities in PDGFRA, IDH1, EGFR, and NF1." Cancer Cell 17 (1): 98-110. https://doi.org/10.1016/j.ccr.2009.12.020.

Vora, Parvez, Chitra Venugopal, Sabra Khalid Salim, Nazanin Tatari, David Bakhshinyan, Mohini Singh, Mathieu Seyfrid, et al. 2020. "The Rational Development of CD133-Targeting Immunotherapies for Glioblastoma." Cell Stem Cell 26 (6): 832-844.e6. https://doi.org/10.1016/j.stem.2020.04.008.

Wajant, Harald. 2016. "Therapeutic Targeting of CD70 and CD27." Expert Opinion on Therapeutic Targets 20 (8): 959-73. https://doi.org/10.1517/14728222.2016.1158812.

Wang, Jiguang, Emanuela Cazzato, Erik Ladewig, Veronique Frattini, Daniel I. S. Rosenbloom, Sakellarios Zairis, Francesco Abate, et al. 2016. "Clonal Evolution of Glioblastoma under Therapy." Nature Genetics 48 (7): 768-76. https://doi.org/10.1038/ng.3590. 
Wang, Qianghu, Baoli Hu, Xin Hu, Hoon Kim, Massimo Squatrito, Lisa Scarpace, Ana C. deCarvalho, et al. 2017. "Tumor Evolution of Glioma Intrinsic Gene Expression Subtype Associates with Immunological Changes in the Microenvironment." Cancer Cell 32 (1): 42-56.e6. https://doi.org/10.1016/j.ccell.2017.06.003.

Wang, Qiong J., Ken-Ichi Hanada, Paul F. Robbins, Yong F. Li, and James C. Yang. 2012. "Distinctive Features of the Differentiated Phenotype and Infiltration of Tumor-Reactive Lymphocytes in Clear Cell Renal Cell Carcinoma." Cancer Research 72 (23): 6119-29. https://doi.org/10.1158/0008-5472.CAN-12-0588.

Winkels, Holger, Svenja Meiler, Esther Smeets, Dirk Lievens, David Engel, Charlotte Spitz, Christina Bürger, et al. 2017. "CD70 Limits Atherosclerosis and Promotes Macrophage Function." Thrombosis and Haemostasis 117 (1): 164-75. https://doi.org/10.1160/TH16-04-0318.

Wischhusen, Jörg, Gundram Jung, Ivan Radovanovic, Christoph Beier, Joachim P. Steinbach, Andreas Rimner, Huatao Huang, et al. 2002. "Identification of CD70Mediated Apoptosis of Immune Effector Cells as a Novel Immune Escape Pathway of Human Glioblastoma." Cancer Research 62 (9): 2592-99.

Xiong, Zujian, Qi Yang, and Xuejun Li. 2020. "Effect of Intra- and Inter-Tumoral Heterogeneity on Molecular Characteristics of Primary IDH-Wild Type Glioblastoma Revealed by Single-Cell Analysis." CNS Neuroscience \& Therapeutics 26 (9): 981-89. https://doi.org/10.1111/cns.13396.

Yang, Z.-Z., D. M. Grote, B. Xiu, S. C. Ziesmer, T. L. Price-Troska, L. S. Hodge, D. M. Yates, A. J. Novak, and S. M. Ansell. 2014. "TGF- $\beta$ Upregulates CD70 Expression and Induces Exhaustion of Effector Memory T Cells in B-Cell NonHodgkin's Lymphoma." Leukemia 28 (9): 1872-84. https://doi.org/10.1038/leu.2014.84.

Yi, Li, Luqing Tong, Tao Li, Long Hai, Iruni Roshanie Abeysekera, Zhennan Tao, Haiwen Ma, et al. 2018. "Bioinformatic Analyses Reveal the Key Pathways and Genes in the CXCR4 Mediated Mesenchymal Subtype of Glioblastoma." Molecular Medicine Reports 18 (1): 741-48.

https://doi.org/10.3892/mmr.2018.9011.

Zeppernick, Felix, Rezvan Ahmadi, Benito Campos, Christine Dictus, Burkhard M. Helmke, Natalia Becker, Peter Lichter, Andreas Unterberg, Bernhard Radlwimmer, and Christel C. Herold-Mende. 2008. "Stem Cell Marker CD133 Affects Clinical Outcome in Glioma Patients." Clinical Cancer Research: An Official Journal of the American Association for Cancer Research 14 (1): 123-29. https://doi.org/10.1158/1078-0432.CCR-07-0932.

Zhang, Cheng, Jun Liu, Jiang F. Zhong, and Xi Zhang. 2017. "Engineering CAR-T Cells." Biomarker Research 5: 22. https://doi.org/10.1186/s40364-017-0102-y.

Zheng, Xiaofeng, Julienne L. Carstens, Jiha Kim, Matthew Scheible, Judith Kaye, Hikaru Sugimoto, Chia-Chin Wu, Valerie S. LeBleu, and Raghu Kalluri. 2015. "Epithelial-to-Mesenchymal Transition Is Dispensable for Metastasis but Induces Chemoresistance in Pancreatic Cancer." Nature 527 (7579): 525-30. https://doi.org/10.1038/nature16064. 
bioRxiv preprint doi: https://doi.org/10.1101/2021.06.02.446670; this version posted June 2, 2021. The copyright holder for this preprint (which

A was not certified by peer review) is the author/funder, who has granted biofBiv a license to display the preprint in perpetuity. It is made available under aCC-BY-NC-ND 4.0 International license.
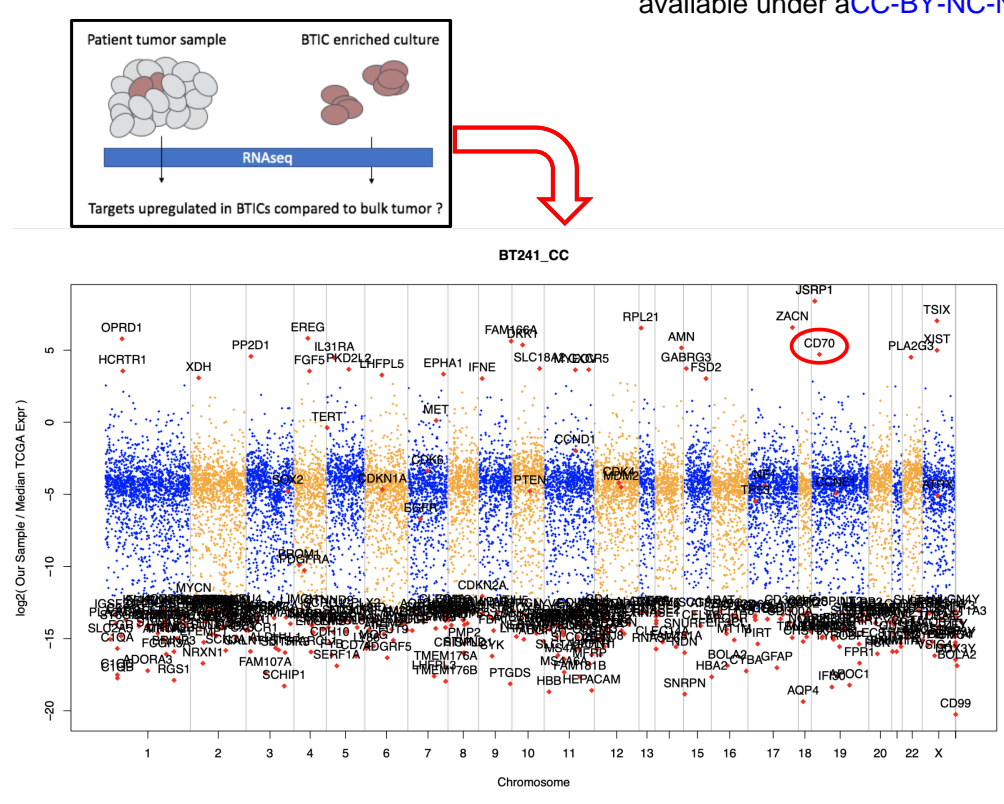

C

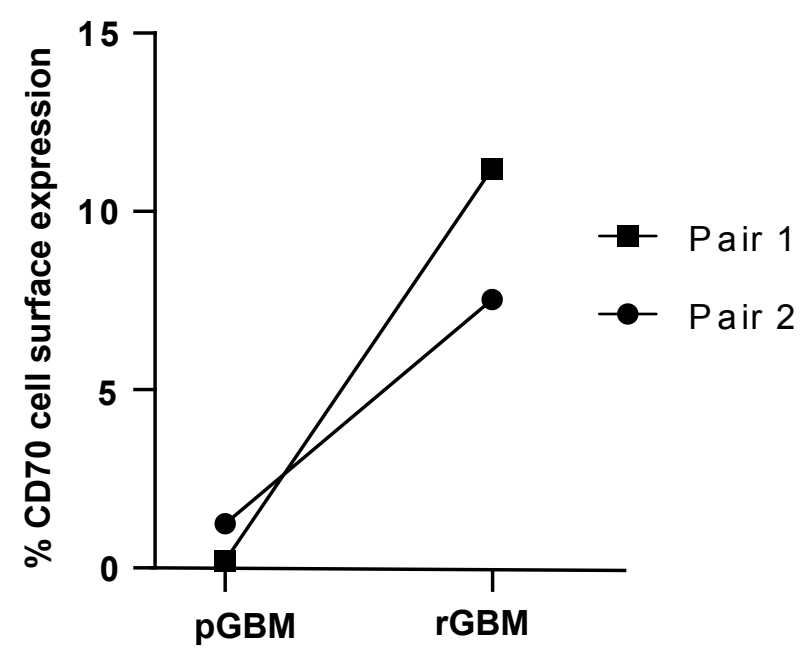

E

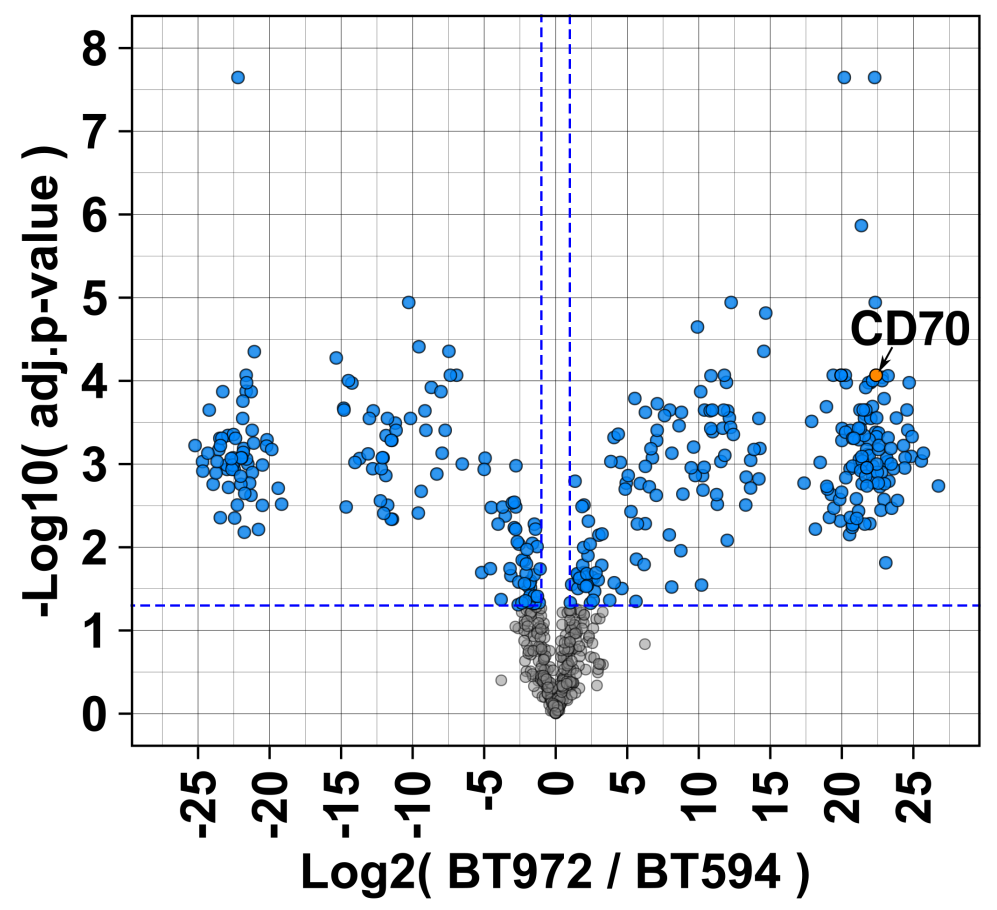

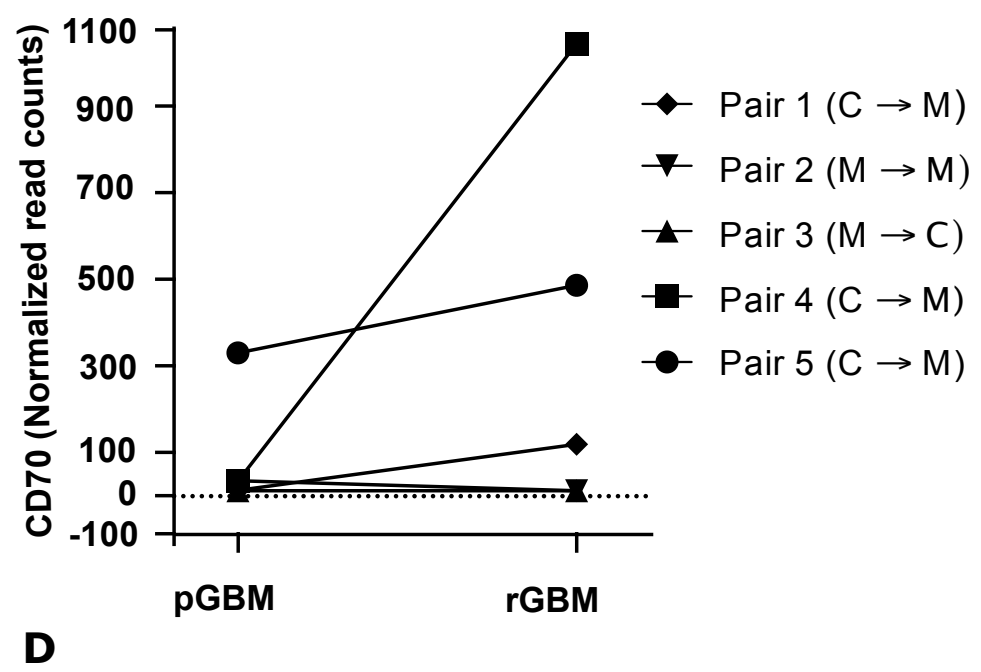

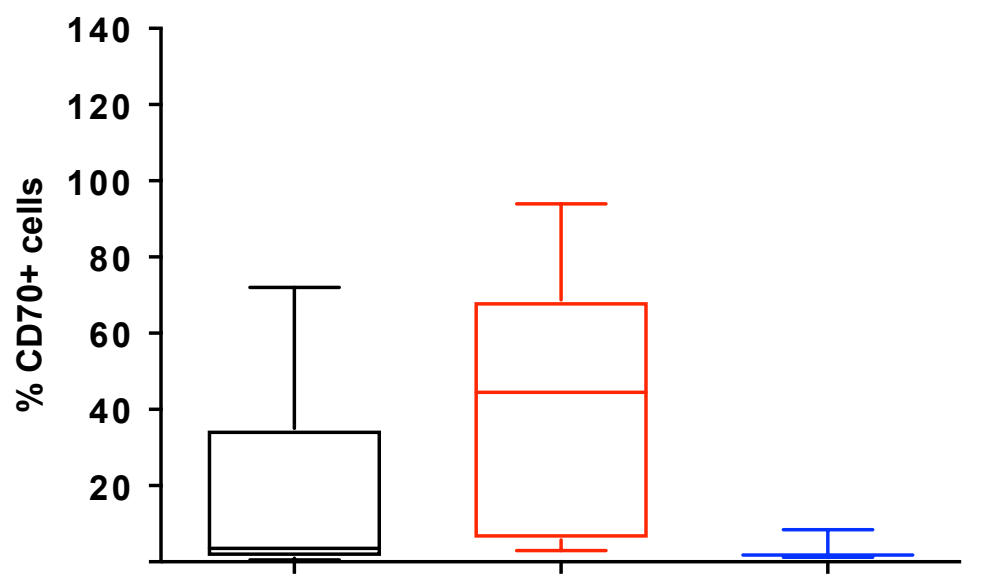

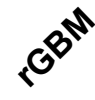

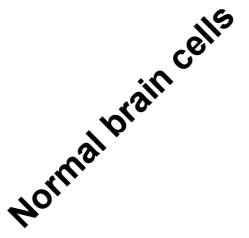

Figure 1 


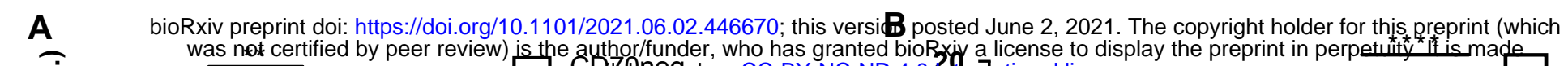

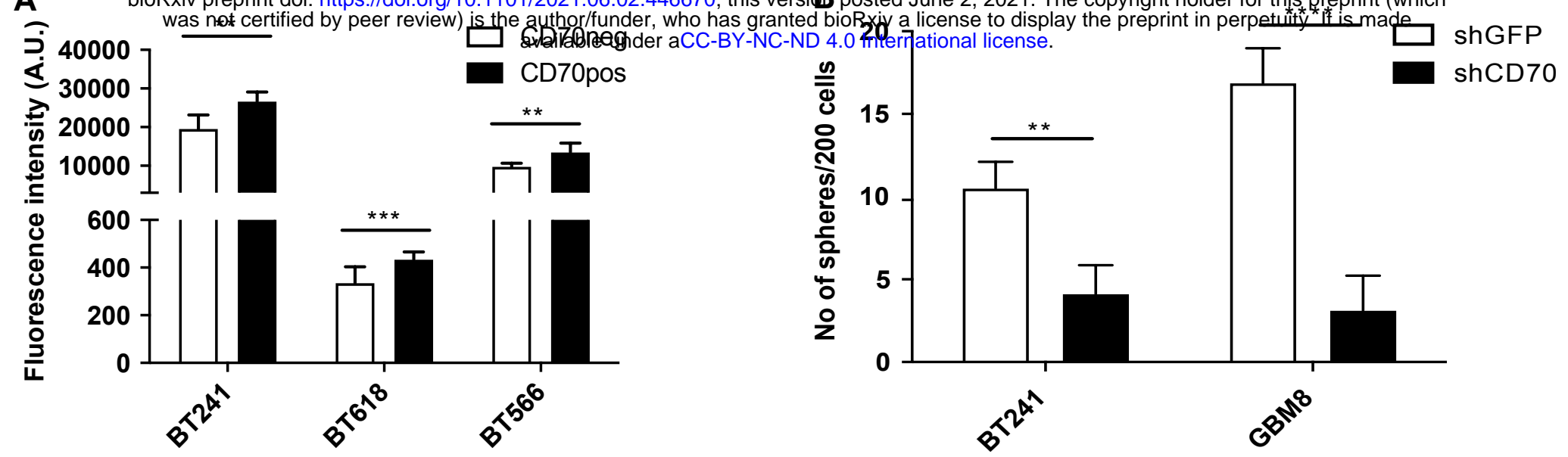

C

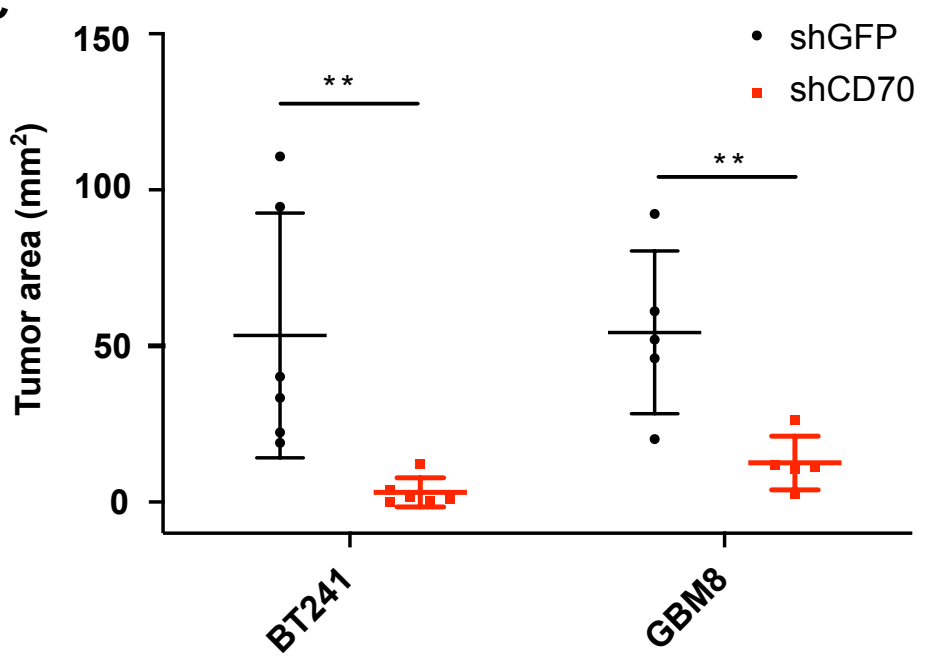

E

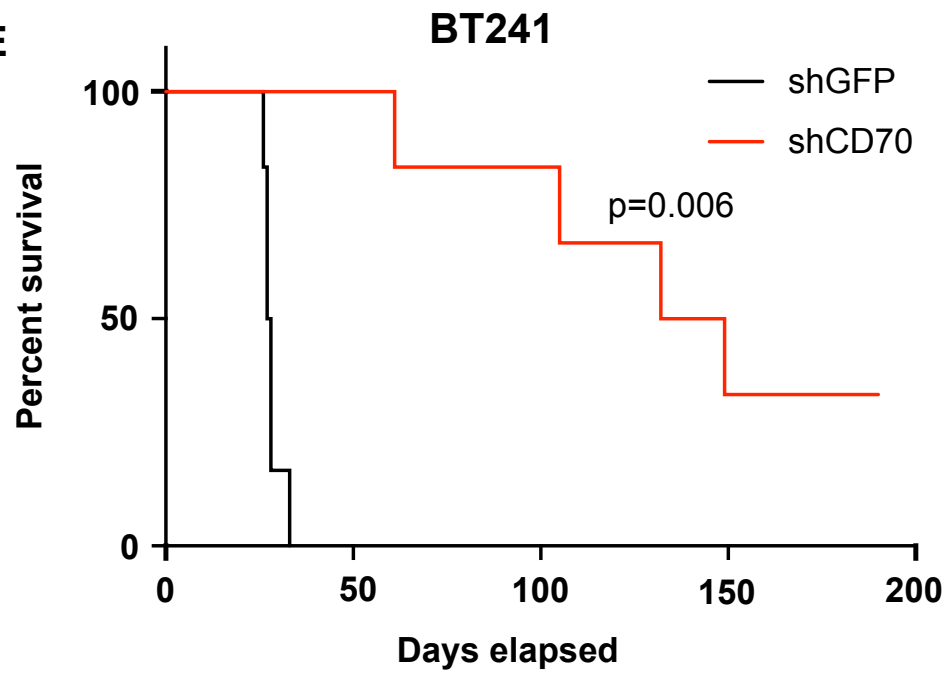

D
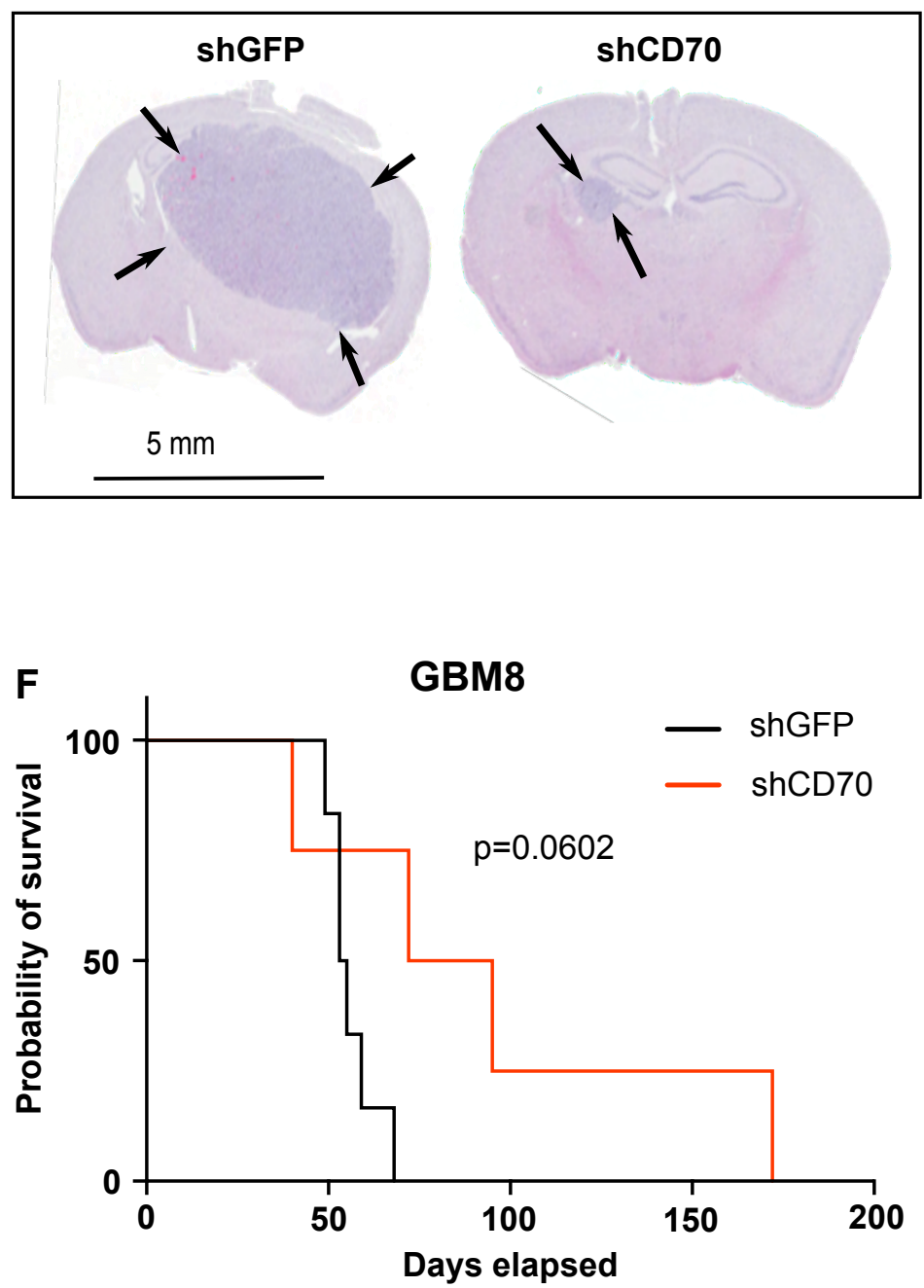

G

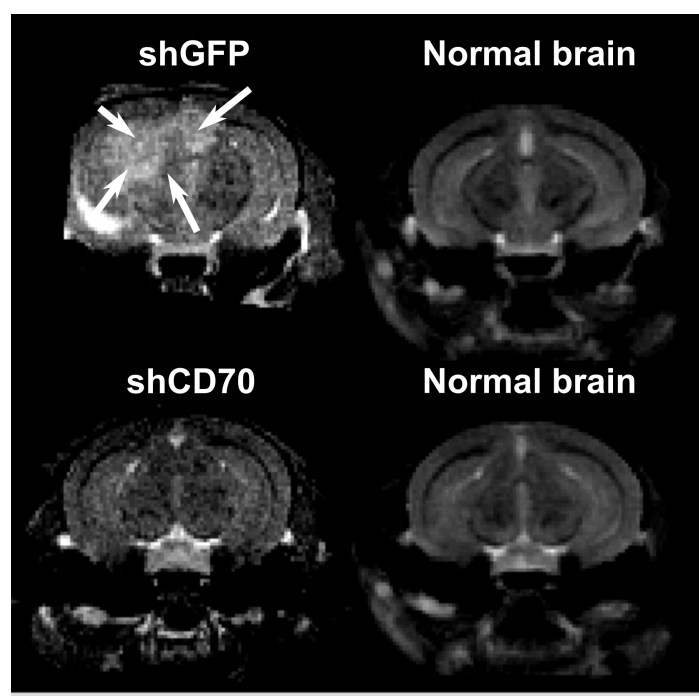


A

$\rightarrow$ he-l-IgG

$\rightarrow$ he-lm-lgG

$\mp$ Commercial standard

B

biokxiv preprint doi: https://doi.org/10.1101/2021.06.02.446670; this version posted Junte 2, 2021. The copyright hotde* for this preprint (which***

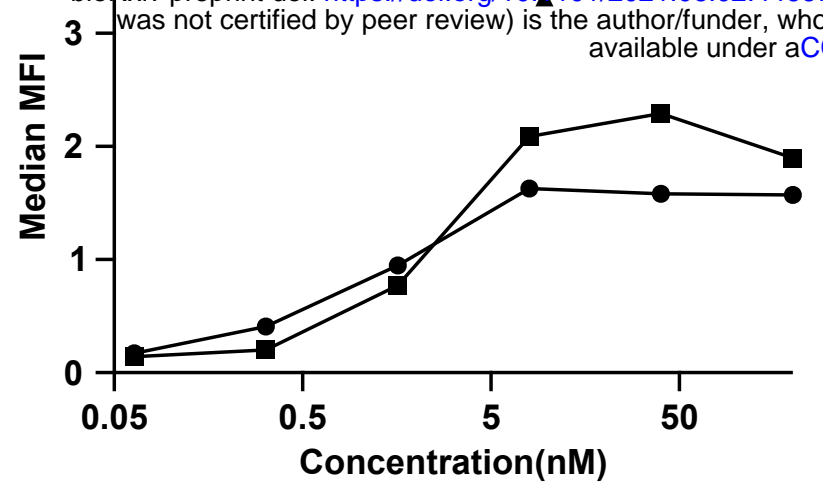

C

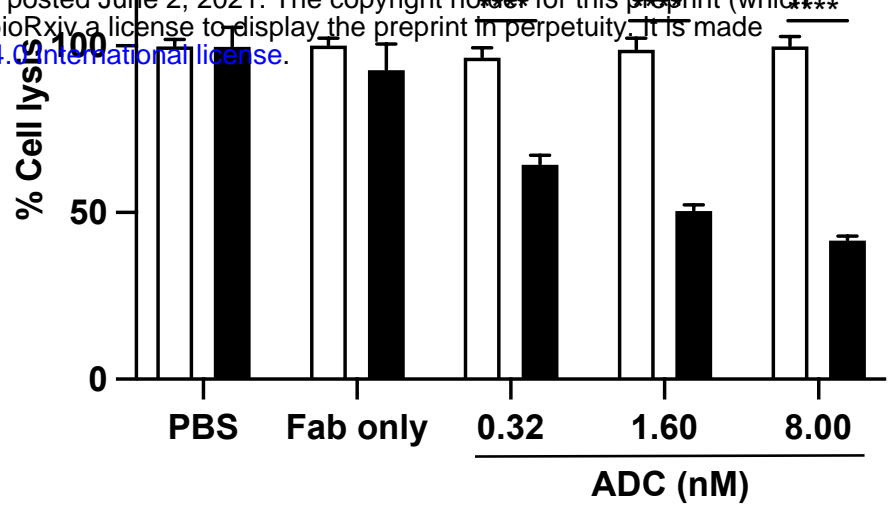

D
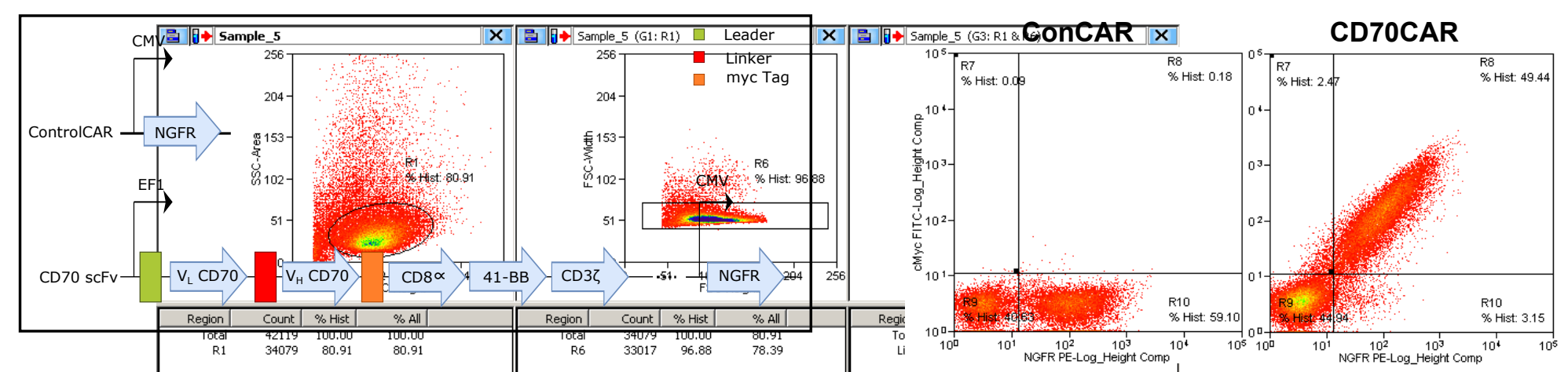

E

F

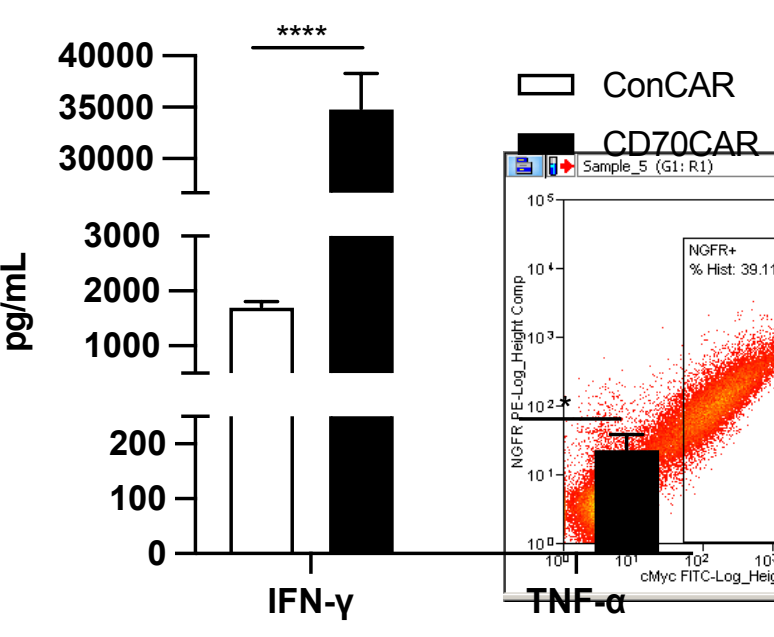

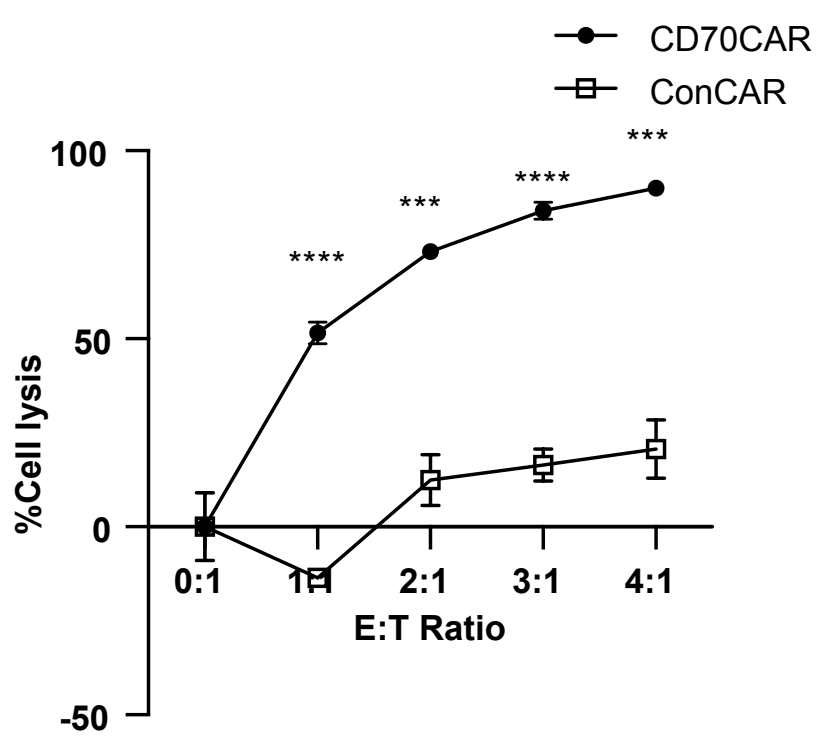


A

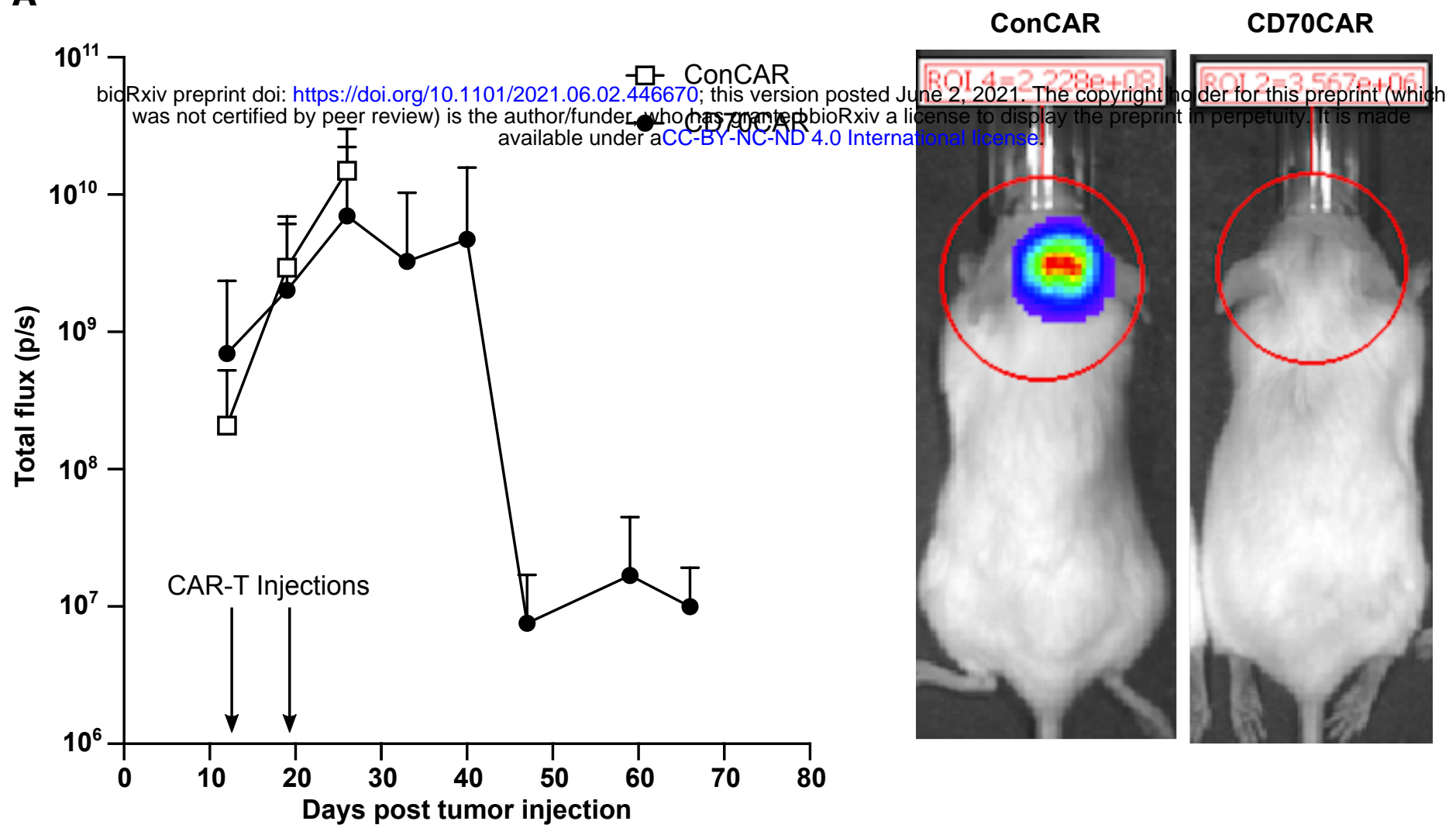

B
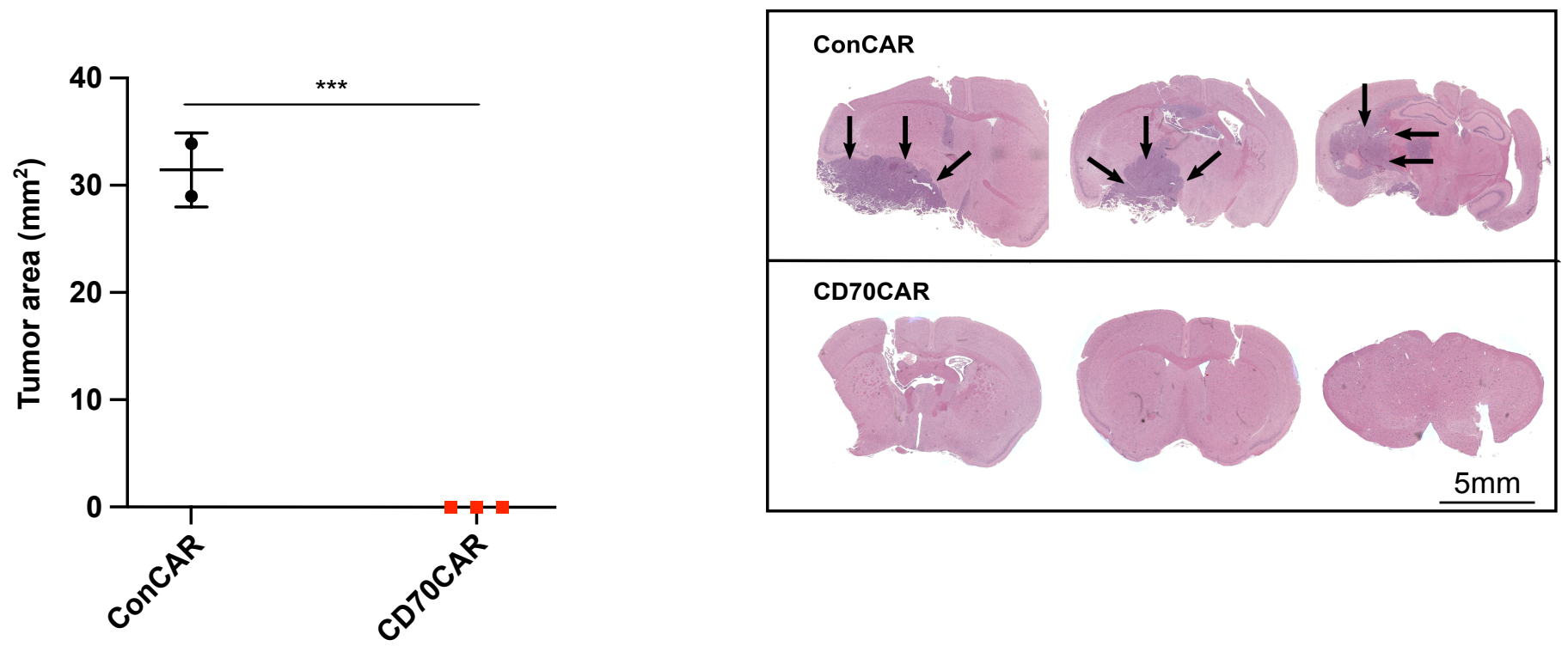

C

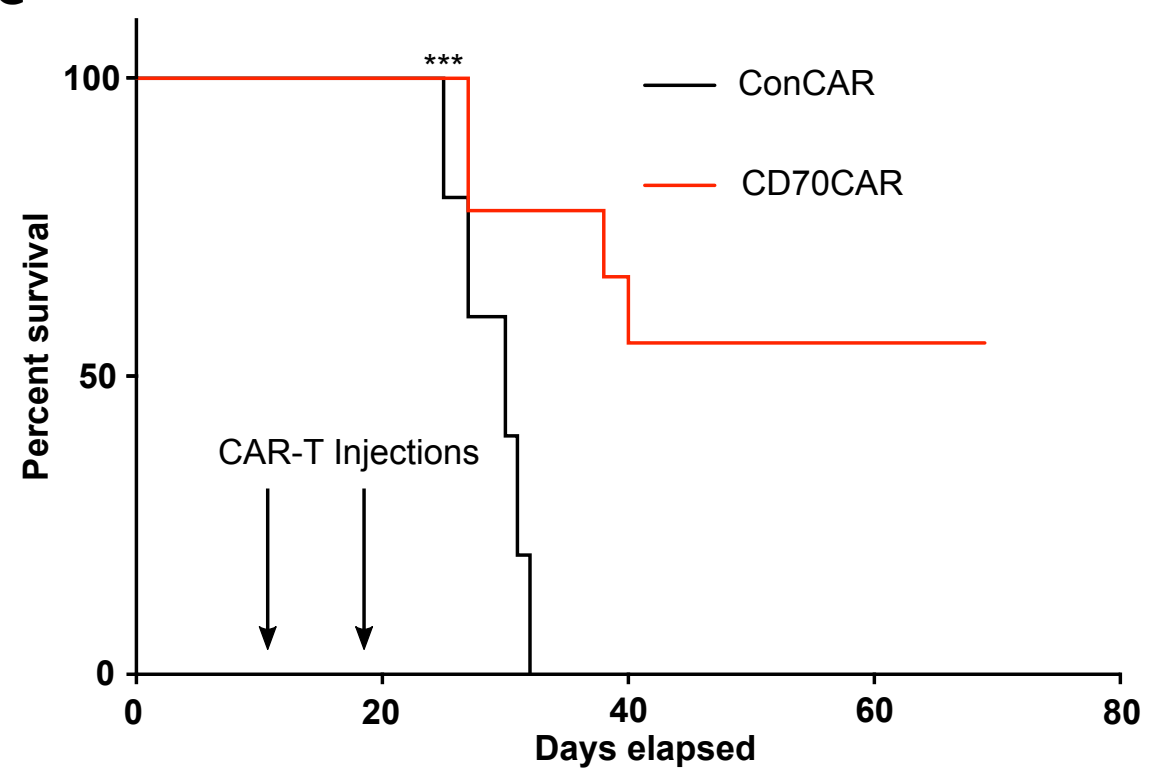


A

B

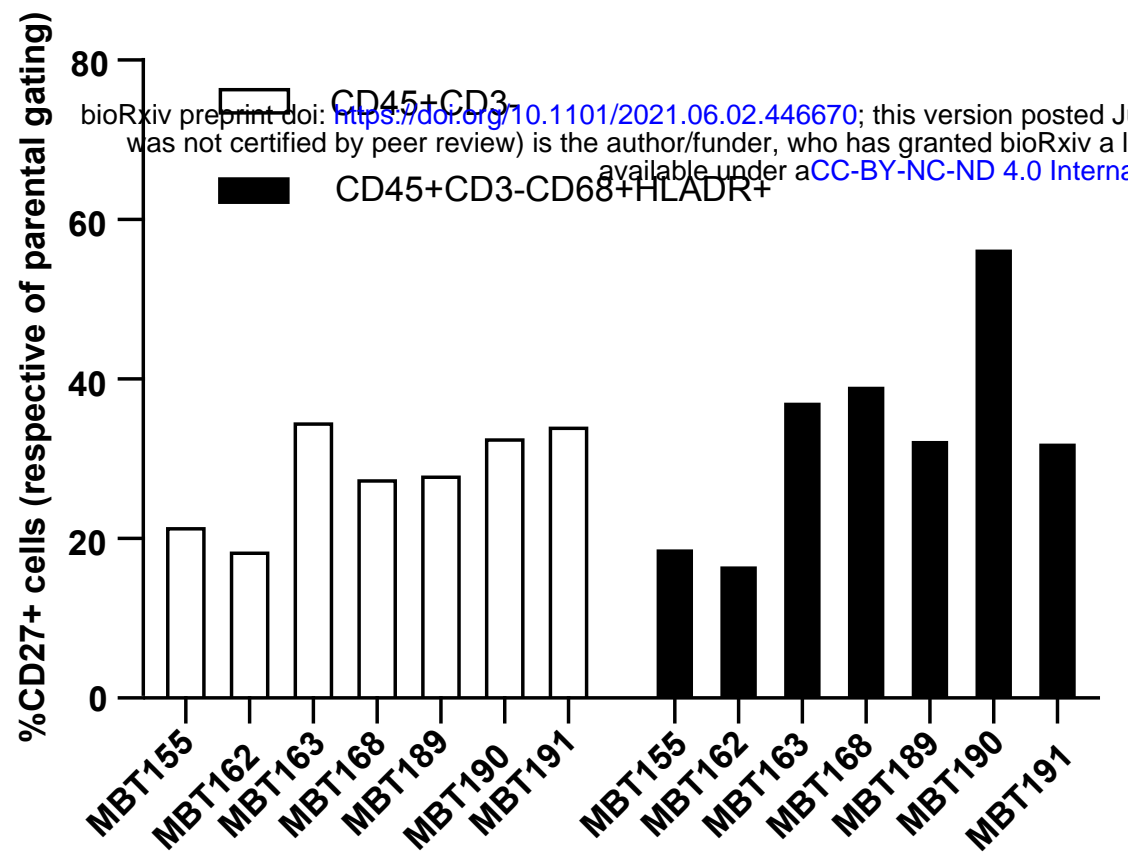

C

\begin{tabular}{|c|c|c|c|c|c|}
\hline \multirow{2}{*}{ Population } & \multicolumn{5}{|c|}{ Days } \\
\cline { 2 - 6 } & $\mathbf{0}$ & $\mathbf{1}$ & $\mathbf{4}$ & $\mathbf{7}$ & $\mathbf{1 1}$ \\
\hline \%CD70 & $<1$ & 6 & 15 & 24 & 28 \\
\hline
\end{tabular}

D

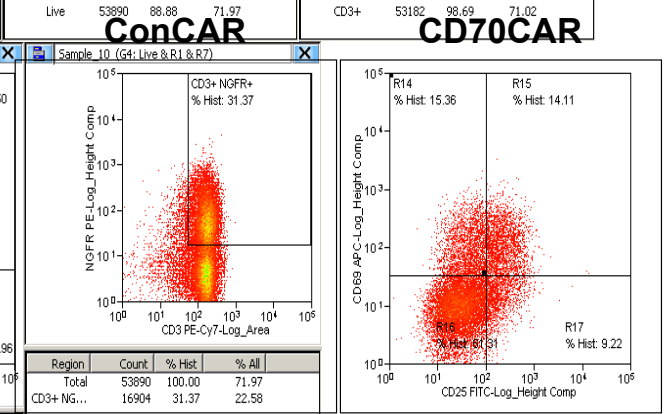

E

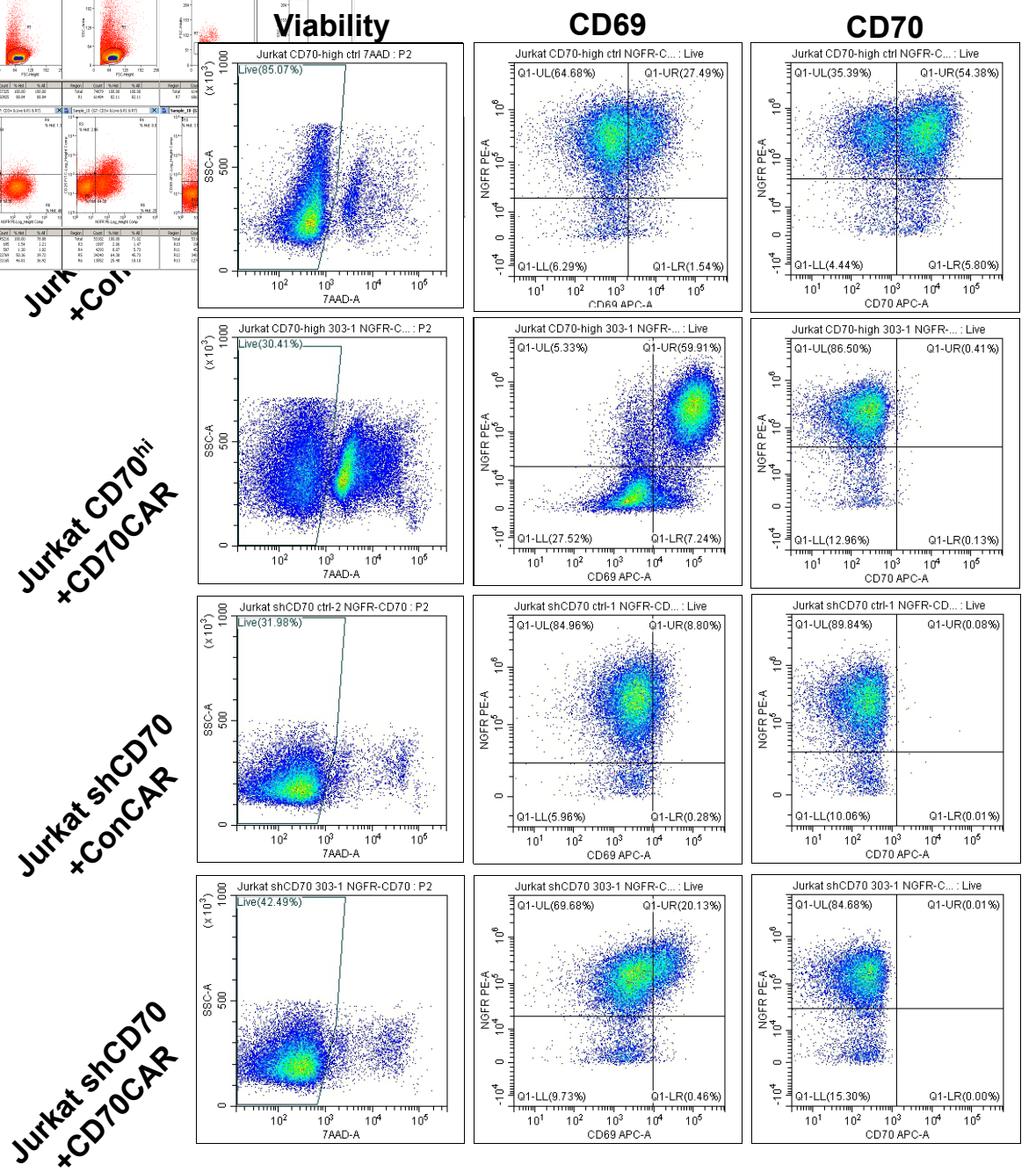




\begin{tabular}{|c|c|c|c|}
\hline Sample & Age & Sex & Primary/Recurrent (P/R)? \\
\hline BT241 & 66 & $\mathrm{~F}$ & $\mathrm{R}$ \\
\hline BT972 & 53 & $\mathrm{M}$ & $\mathrm{R}$ \\
\hline BT594 & 50 & $\mathrm{M}$ & $\mathrm{P}$ \\
\hline BT618 & 67 & $\mathrm{~F}$ & $\mathrm{R}$ \\
\hline BT566 & 55 & $\mathrm{~F}$ & $\mathrm{R}$ \\
\hline BT155 & 66 & $\mathrm{~F}$ & $\mathrm{P}$ \\
\hline BT162 & 57 & $\mathrm{~F}$ & $\mathrm{P}$ \\
\hline BT163 & 56 & $\mathrm{M}$ & $\mathrm{P}$ \\
\hline BT168 & 46 & $\mathrm{~F}$ & $\mathrm{P}$ \\
\hline BT189 & 67 & $\mathrm{~F}$ & $\mathrm{P}$ \\
\hline MBT190 & 27 & $\mathrm{~F}$ & $\mathrm{R}$ \\
\hline BT191 & 50 & $\mathrm{~F}$ & $\mathrm{P}$ \\
\hline BT956 & 70 & $\mathrm{~F}$ & $\mathrm{P}$ \\
\hline BT698 & 57 & $\mathrm{~F}$ & $\mathrm{P}$ \\
\hline MBT225 & 27 & $\mathrm{M}$ & $\mathrm{R}$ \\
\hline MBT173 & 69 & $\mathrm{~F}$ & $\mathrm{R}$ \\
\hline BT566 & 55 & $\mathrm{~F}$ & $\mathrm{R}$ \\
\hline MBT103 & 52 & $\mathrm{~F}$ & $\mathrm{P}$ \\
\hline MBT121 & 67 & $\mathrm{~F}$ & $\mathrm{P}$ \\
\hline BT458 & 81 & $\mathrm{M}$ & $\mathrm{P}$ \\
\hline BT428 & 63 & $\mathrm{~F}$ & $\mathrm{P}$ \\
\hline BT954 & 65 & $\mathrm{M}$ & $\mathrm{P}$ \\
\hline BT799 & 77 & $\mathrm{~F}$ & $\mathrm{P}$ \\
\hline MBT63 & 68 & $\mathrm{~F}$ & $\mathrm{P}$ \\
\hline MBT27 & 56 & $\mathrm{M}$ & $\mathrm{P}$ \\
\hline BT935 & 53 & $\mathrm{~F}$ & $\mathrm{P}$ \\
\hline BT459 & 60 & $\mathrm{M}$ & $\mathrm{P}$ \\
\hline BT778 & 54 & $\mathrm{~F}$ & $\mathrm{P}$ \\
\hline
\end{tabular}


bioRxiv preprint doi: https://doi org/10.1101/2021.06.02.446670; this version posted June 2, 2021. The copyright holder for this preprint (which was not certified by peer review) is the author/funder, who has granted bioRxiv a license to display the preprint in perpetuity. It is made available under aCC-BY-NC-ND 4.0 International license.

\begin{tabular}{|c|c|c|c|c|c|c|}
\hline \multirow{2}{*}{ Ensembl ID } & \multirow{2}{*}{$\begin{array}{c}\text { Gene } \\
\text { symbol }\end{array}$} & \multicolumn{3}{|c|}{ Log (FC) } & \multicolumn{2}{c|}{$\begin{array}{c}\text { Combined Differential } \\
\text { Gene Expression }\end{array}$} \\
\cline { 3 - 7 } & & BT241 & GBM8 & GBM4 & Log (FC) & P-value \\
\hline ENSG00000175592 & FOSL1 & -3.0876903 & -2.9685466 & -1.4827605 & -1.3302582 & 0.002764603 \\
\hline ENSG00000205927 & OLIG2 & 0.99188628 & 1.51882472 & 2.02962725 & 0.95394172 & 0.027641632 \\
\hline ENSG00000112715 & VEGFA & -1.079047 & -2.993489 & -0.2838893 & -0.8847545 & 0.040829495 \\
\hline ENSG00000170558 & CDH2 & -1.8132171 & -0.4377818 & -1.1326002 & -0.633147 & 0.148275349 \\
\hline ENSG00000011422 & PLAUR & -2.0416545 & -1.1341974 & -2.2947466 & -0.4965695 & 0.206493437 \\
\hline ENSG00000121966 & CXCR4 & -1.5615955 & -2.8821719 & -1.3521936 & -0.361023 & 1 \\
\hline ENSG00000026508 & CD44 & -0.6256976 & -0.0583328 & -1.5159146 & -0.3010297 & 0.482572636 \\
\hline ENSG00000181449 & SOX2 & -0.0175167 & -0.8038392 & -0.905513 & -0.3279201 & 0.417025332 \\
\hline
\end{tabular}

